\title{
Phase diagram evolution at finite coupling in strong coupling lattice QCD
}

\section{$\operatorname{AUTHOR}(\mathrm{S}):$}

Miura, Kohtaroh; Nakano, Takashi Z:; Ohnishi, Akira; Kawamoto, Noboru

\section{CITATION:}

Miura, Kohtaroh ... [et al]. Phase diagram evolution at finite coupling in strong coupling lattice QCD. Physical Review D - Particles, Fields, Gravitation and Cosmology 2009, 80(7): 074034.

ISSUE DATE:

2009-10

URL:

http://hdl.handle.net/2433/89516

RIGHT:

c 2009 The American Physical Society. 
PHYSICAL REVIEW D 80, 074034 (2009)

\title{
Phase diagram evolution at finite coupling in strong coupling lattice QCD
}

\author{
Kohtaroh Miura, ${ }^{1, *}$ Takashi Z. Nakano, ${ }^{2}$ Akira Ohnishi, ${ }^{1}$ and Noboru Kawamoto ${ }^{3}$ \\ ${ }^{1}$ Yukawa Institute for Theoretical Physics, Kyoto University, Kyoto 606-8502, Japan \\ ${ }^{2}$ Department of Physics, Faculty of Science, Kyoto University, Kyoto 606-8502, Japan \\ ${ }^{3}$ Department of Physics, Faculty of Science, Hokkaido University, Sapporo 060-0810, Japan
}

(Received 5 August 2009; published 28 October 2009)

\begin{abstract}
We investigate the chiral phase transition in the strong coupling lattice QCD at finite temperature and density with finite coupling effects. We adopt one species of staggered fermion, and develop an analytic formulation based on strong coupling and cluster expansions. We derive the effective potential as a function of two order parameters, the chiral condensate $\sigma$ and the quark number density $\rho_{q}$, in a selfconsistent treatment of the next-to-leading order (NLO) effective action terms. NLO contributions lead to modifications of quark mass, chemical potential, and the quark wave function renormalization factor. While the ratio $\mu_{c}(T=0) / T_{c}(\mu=0)$ is too small in the strong coupling limit, it is found to increase as $\beta=2 N_{c} / g^{2}$ increases. The critical point is found to move in the lower $T$ direction as $\beta$ increases. Since the vector interaction induced by $\rho_{q}$ is shown to grow as $\beta$, the present trend is consistent with the results in Nambu-Jona-Lasinio models. The interplay between two order parameters leads to the existence of partially chiral restored matter, where effective chemical potential is automatically adjusted to the quark excitation energy.
\end{abstract}

DOI: 10.1103/PhysRevD.80.074034

\section{INTRODUCTION}

Exploring the chiral phase transition and its phase diagram in quantum chromodynamics (QCD) is one of the most challenging problems in quark hadron physics. The chiral phase transition may really happen in compact astrophysical phenomena such as the early Universe and compact stars, and can be investigated in heavy-ion collision experiments. The large magnitude of the elliptic flow parameter observed in the relativistic heavy-ion collider (RHIC) experiments indicates the formation of strongly interacting quark-gluon plasma [1-4] at high temperature. The future experiments at FAIR and in low-energy programs at RHIC are expected to provide new discoveries in the phase diagram investigations.

The most rigorous and reliable framework to investigate the QCD phase transition would be the lattice QCD Monte Carlo (MC) simulations. In the high temperature and low density region, the lattice $\mathrm{MC}$ can provide the quantitative predictions, and the critical temperature is estimated to be $T_{c} \simeq(160-190) \mathrm{MeV}[5,6]$. In comparison, the lattice MC simulations do not work well in the high baryon density region because of the notorious sign problem of the Dirac determinant. Many ideas have been proposed to overcome this problem [7], for example, the Taylor expansion around $\mu=0$ [8], analytic continuation [9,10], canonical ensemble method [11], improved reweighting method [12], and the density of states method [13]. It has become possible to access the relatively small density region $\mu / T \lesssim 1.0[6,7]$. One of the interesting objects is the critical end point (CEP) [14]. Recent works

\footnotetext{
*miura@yukawa.kyoto-u.ac.jp
}

PACS numbers: 11.15.Me, 12.38.Gc, 11.10.Wx, 25.75.Nq

indicate that the CEP may locate in the region $\mu / T \geq 1.0$ [15-17], while de Forcrand and Philipsen suggest that the CEP might not exist [18]. The larger chemical potential region is still under debate, and alternative methods are necessary to reveal the whole structure of the phase diagram.

In the phase diagram investigation, the strong coupling lattice QCD (SC-LQCD), the lattice QCD formulation based on the expansion of the action in the power series of the inverse bare coupling squared $\left(1 / g^{2}\right)$, is an interesting approach, since the sign problem can be weakened or avoided. The SC-LQCD was applied first to the confinement study in pure Yang-Mills theories [19-21]. Wilson suggested the confinement mechanism in an analytic study of the strong coupling limit (SCL) of lattice QCD [21]. Creutz showed that the $\beta=2 N_{c} / g^{2}$ dependence of the lattice spacing $a$ in the MC simulation smoothly connects the strong coupling behavior and the continuum spacetime scaling behavior [22]. By using the character expansion technique, Münster demonstrated that the pure Yang-Mills SC-LQCD with high order corrections could explain the above MC results [23]. The scaling behavior in MC simulations indicates that the confinement is actually realized in the continuum spacetime, and the success of SC-LQCD suggests that the scaling region would be accessible in $\mathrm{SC}$ LQCD within the conversion radius, which is shown to be finite in pure Yang-Mills theories [24]. We may expect that the scaling and convergent properties are also kept with fermions. Then it would be possible that the SC-LQCD could provide useful results on the phase diagram in the whole region of the $T-\mu$ plane.

The SC-LQCD with fermions has a long history of chiral symmetry studies for more than 20 years [25-61], and 
many theoretical tools have been developed; the large dimensional or $1 / d(d=$ spatial or spacetime dimension) expansion [30], the finite temperature treatments in the Polyakov gauge [34] and in the temporal gauge [36], the finite quark chemical potential effect [35] with the help of the lattice chemical potential [62]. The analytic expression of the SCL effective potential has been derived at finite $T$ [34,36] or at finite $\mu$ [35]. In the 1990s, phase diagram studies met some successes [37-39] based on the SCLQCD effective action [36]. We also find several works on the Polyakov loop [63] based on SC-LQCD, and the functional form of the effective potential in SC-LQCD has provided basic ingredients in the SC-LQCD related models [64-66] and the Nambu-Jona-Lasinio model with Polyakov loop (PNJL) [67].

Based on these successes, the SC-LQCD is recently revisited and expected to provide an instructive guide to QCD under extreme conditions. The pure Yang-Mills SCLQCD [23] is extended to finite $T$ [68], and the shear viscosity below the deconfinement transition temperature is also studied [69]. In color $S U(2)$ QCD, the interplay between the diquark condensate $\Delta$ and the chiral condensate $\sigma$ is investigated at finite $T$ and $\mu$ [40]. The diquark effect is also investigated for color $S U(3)$ at zero temperature [41]. We find remarkable developments in the $S U\left(N_{c}=3\right)$ phase diagram investigations [42-44,51], where a "naïve" structure of the phase diagram with first and second transition lines separated by a tricritical point (TCP) is obtained in the strong coupling limit with zero quark masses. With nonzero quark masses, TCP becomes a CEP, whose discovery is one of the physics goals in lowenergy programs at RHIC.

In order to discuss the chiral symmetry on the lattice, the SC-LQCD has been developed in several fermion formalisms. We find some pioneering works based on the staggered [25,26], the Wilson [25,28], and the naïve [29] fermions. The domain-wall [70] and the overlap [71] fermion provide modern formulation of the lattice chiral symmetry, and some SC-LQCD based investigations are found in [58,59] (domain-wall) and [60,61] (overlap). In the present work, we adopt one species $\left(n_{f}=1\right)$ of (unrooted) staggered fermion. Its simple realization of the chiral symmetry on the lattice $[25,72,73]$ is useful to develop analytic formulations. It has been theoretically suggested [32,74] and numerically established [75] that the unrooted staggered QCD is equivalent to the four flavor $\left(N_{f}=4\right)$ QCD with degenerate masses in the continuum limit.

In this paper, we investigate the phase diagram evolution with finite coupling effects. We employ one species of (unrooted) staggered fermion, and take account of the next-to-leading order [NLO, $\left.\mathcal{O}\left(1 / g^{2}\right)\right]$ terms in the strong coupling expansion. We concentrate on the leading order of the large dimensional $(1 / d)$ expansion [30] for simplicity. The gluon field is evaluated in the Polyakov gauge [34] with respect for the finite temperature $T$ effects, and the finite density effects are introduced via the quark chemical potential $\mu$ on the lattice [62]. In these setups, we derive an analytic expression of the effective potential in the mean field approximation. In particular, the following points are newly developed. First, we introduce the NLO effective action terms through the systematic cluster expansion. Second, we evaluate the NLO effective action by using a recently developed extended Hubbard-Stratonovich (EHS) transformation $[45,46]$. As a result, several auxiliary fields including the chiral condensate $\sigma$ are introduced on the same footing, and the NLO effects are self-consistently evaluated. In particular, we find that the quark number density naturally appears as an order parameter, whose self-consistent solution in equilibrium plays essential roles in the large $\mu$ region. This point would be an advantage to the previous works with NLO effects [36-39]. Third, we discuss the evolution of the first and second order transition lines and the critical point with $\beta=2 N_{c} / g^{2}$. The finite coupling effects on the critical point have not been investigated before. Fourth, the NLO contribution is expressed as modifications of the constituent quark mass, chemical potential, and the quark wave function renormalization factor. Hence the mechanism of the phase diagram evolution becomes clear.

While we are working based on the strong coupling expansion with NLO effects, we expect the present work would give a valuable picture in understanding the QCD phase diagram in the real world through the relation with the MC simulations. Since the SC-LQCD is based on the same formulation as lattice $\mathrm{MC}$ simulations, its results should be consistent with $\mathrm{MC}$ results as long as the applied approximations are valid. This speculation is supported by previous works on the hadron mass spectrum [27,30,31]. Very recently, the structure of the phase diagram suggested in SC-LQCD is qualitatively confirmed by a lattice MC simulation in the strong coupling limit [51] based on a monomer-dimer-polymer formulation [53]. Thus the phase diagram in the strong coupling limit is established from both sides of analytic and numerical studies, and provides a good starting point to explore the true phase diagram by evaluating finite coupling effects in the strong coupling expansions. We find MC studies using one species of unrooted staggered quarks, and the results around $\beta \sim 5$ have been extensively discussed $[10,76]$. In order to compare the SC-LQCD results with those in MC simulations, we discuss the results in the region $\beta \leq 6$ expecting that these $\beta$ values are within the conversion radius.

Although the number of flavors $\left(N_{f}=4 n_{f}=4\right)$ used in the present work is different from the real world $\left(N_{f}=\right.$ $2+1$ ), we could provide valuable results for the phase diagram investigations. Flavor dependence of the phase diagrams at strong coupling has been studied by using several species $\left(n_{f}=2,3\right)$ of staggered fermions $[38,43]$, and we find that the phase diagrams with $n_{f}=2$ and 3 are 
qualitatively the same as that with $n_{f}=1$. The critical chemical potentials at $T=0\left(\mu_{c, T=0}\right)$ are found to be almost the same. The critical temperatures at $\mu=0$ $\left(T_{c, \mu=0}\right)$ are found to be around $1.2[38,43]$ and $1.06[38]$ for $n_{f}=2$ and 3 , respectively. These values differ from the result of $n_{f}=1\left(T_{c, \mu=0}=5 / 3\right)$ by $30-40 \%$, but the obtained phase diagram structure is very similar. The flavor dependence of the phase diagram is found to be moderate also in the continuum region. In Ref. [10], the small $\mu$ region of phase diagram is investigated by using $\mathrm{MC}$ simulations with four flavor staggered quarks, and the results are compared with two flavor results [9]. The difference between the phase boundaries in two and four flavor cases is at most $7 \%$ within a region $N_{c} \mu<$ $500 \mathrm{MeV}$ [10]. Thus, a "shape" of the phase boundary may not be crucially affected by the flavor effects. It should be noted that the number of flavors is important to some of the key features of the phase diagram, such as the order of the phase transition and the position of the critical point.

The organization of this paper is as follows. In Sec. II, we provide a brief review on the strong coupling $\left(1 / g^{2}\right)$, the large dimensional $(1 / d)$ and cluster expansions, and derive the effective action including the $\mathcal{O}\left(1 / g^{2}, 1 / d^{0}\right)$ effects. In Sec. III, we derive an analytic expression of the effective potential. In Sec. IV, we investigate the phase diagram evolution with $\beta$, and focus on the mechanisms of the critical temperature and chemical potential modifications. The "partially chiral restored (PCR) matter" is found to appear in the high density region, and we also discuss its origin. Finally we summarize our work in Sec. V. All through this paper, we use the lattice unit $a=$ 1 , and physical values are normalized by $a$.

\section{EFFECTIVE ACTION}

\section{A. Lattice QCD action}

We start from the lattice QCD action and the partition function with one species of staggered fermion $\chi$ with a quark mass $m_{0}$. Gluons are represented by the temporal link $\left(U_{0}\right)$ and spatial link $\left(U_{j}, j=1,2, \cdots, d\right)$ variables,

$$
\begin{gathered}
Z=\int \mathcal{D}\left[\chi, \bar{\chi}, U_{0}, U_{j}\right] \exp \left[-S_{\mathrm{LQCD}}\right], \\
S_{\mathrm{LQCD}}=S_{F}^{(\tau)}+\sum_{x} m_{0} M_{x}+S_{F}^{(s)}+S_{G}, \\
S_{F}^{(\tau)}=\frac{1}{2} \sum_{x}\left[e^{\mu} \bar{\chi}_{x} U_{0} \chi_{x+\hat{0}}-e^{-\mu} \bar{\chi}_{x+\hat{0}} U_{0}^{\dagger} \chi_{x}\right] \\
\equiv \frac{1}{2} \sum_{x}\left[V_{x}^{+}(\mu)-V_{x}^{-}(\mu)\right], \\
S_{F}^{(s)}=\sum_{x, j} \frac{\eta_{j, x}}{2}\left[\bar{\chi}_{x} U_{j, x} \chi_{x+\hat{j}}-(\text { H.c. })\right] \equiv \sum_{x, j} s_{j, x},
\end{gathered}
$$

$$
S_{G}=\frac{2 N_{c}}{g^{2}} \sum_{P}\left[1-\frac{1}{2 N_{c}}\left[U_{P}+U_{P}^{\dagger}\right]\right] .
$$

Here the trace of the plaquette $U_{P}$ is defined as

$$
U_{P=\mu \nu, x}=\operatorname{tr}_{c}\left[U_{\mu, x} U_{\nu, x+\hat{\mu}} U_{\mu, x+\hat{\nu}}^{\dagger} U_{\nu, x}^{\dagger}\right] .
$$

In this action, $M_{x}$ denotes the mesonic composite, $M_{x}=$ $\bar{\chi}_{x} \chi_{x}$, and we have defined two other mesonic composites, $V^{ \pm}$, which contain the temporal link variables. Sums over color indices are assumed. Quark chemical potential on the lattice $(\mu)$ is introduced as a weight of the temporal hopping in the exponential form [62], and the staggered phase factor $\eta_{j, x}=(-1)^{x_{0}+\cdots+x_{j-1}}$ in the spatial action is related to the Dirac's $\gamma$ matrices [25,32]. By using a $\gamma_{5}$-related factor $\epsilon_{x}=(-1)^{x_{0}+\cdots+x_{d}}$, a staggered chiral transformation is given as $\chi_{x} \rightarrow e^{i \theta \epsilon_{x}} \chi_{x}[25,72,73]$. The lattice kinetic action $S_{F}^{(\tau, s)}$ is invariant under this chiral transformation in the chiral limit $m_{0} \rightarrow 0$.

Throughout the paper, we consider the color $S U\left(N_{c}=\right.$ $3)$ case in $3+1$ dimensions $(d=3)$. Temporal and spatial lattice sizes are $N_{\tau}$ and $L$, respectively. While $T=1 / N_{\tau}$ takes discrete values, we consider $T$ as a continuous valued temperature. We take account of finite $T$ effects by imposing periodic and antiperiodic boundary conditions on link variables and quarks, respectively. We take the static and diagonalized gauge (called the Polyakov gauge) for temporal link variables with respect for the periodicity [34].

\section{B. Spatial link integral in the strong coupling limit}

In the finite temperature $(T)$ treatment, we obtain the effective action of quarks $(\chi, \bar{\chi})$ and temporal link variable $\left(U_{0}\right)$ by integrating out the spatial link variables $\left(U_{j}\right)$. We shall evaluate the spatial partition function,

$$
Z^{(s)}=\int \mathcal{D} U_{j} \exp \left[-S_{F}^{(s)}-S_{G}\right]
$$

and integrate out the spatial link variables $\left(U_{j}\right)$. In the strong coupling region $(g \gg 1)$, we can treat the plaquette action term $\left(S_{G} \propto 1 / g^{2}\right)$ through the expansion in the power series of $1 / g^{2}$ (strong coupling expansion).

In the SCL, we can omit $S_{G}$, and the spatial partition function is decomposed into that on each link,

$$
Z_{\mathrm{SCL}}^{(s)}=\int \mathcal{D} U_{j} e^{-S_{F}^{(s)}}=\prod_{j, x}\left[\int d U_{j} \exp \left[-s_{j, x}\right]\right] .
$$

We can carry out the $U_{j}$ integral on each link $(j, x)$ by utilizing the one-link $S U\left(N_{c}\right)$ group integral formulas [77],

$$
\begin{gathered}
\int d U U_{a b} U_{c d}^{\dagger}=\frac{1}{N_{c}} \delta_{a d} \delta_{b c}, \\
\int d U U_{a b} U_{c d} \cdots U_{e f}=\frac{1}{N_{c} !} \epsilon_{a c \cdots e} \epsilon_{b d \cdots f},
\end{gathered}
$$

and other higher order integral formulas. The spatial part of 
MIURA et al.

TABLE I. The coefficients of the hadronic composites in the effective action at the strong coupling limit. Detailed explanation to calculate these coefficients are found in Ref. [77].

\begin{tabular}{lc}
\hline \hline Coefficients & Values $\left(N_{c}=3\right)$ \\
\hline$A_{1}$ & $-1 /\left(4 N_{c}\right)$ \\
$A_{2}$ & $-\left(N_{c}^{2} \cdot\left(N_{c}-2\right) !-N_{c} !\right) /\left(32 \cdot N_{c}^{2} \cdot N_{c} !\right)$ \\
$A_{3}$ & $-\left(2 \cdot N_{c} !-N_{c}^{3} \cdot\left(N_{c}-2\right) !\right) /\left(128 \cdot N_{c}^{4} \cdot N_{c} !\right)$ \\
$A_{j, x}$ & $-(-1)^{N_{c}\left(N_{c}-1\right) / 2} \eta_{j, x}^{N_{c}} / 2^{N_{c}}$ \\
\hline \hline
\end{tabular}

the hadronic effective action density is obtained as [26],

$$
\int d U_{j} \exp \left[-s_{j, x}\right]=\exp \left[-s_{j, x}^{(\text {eff })}\right]
$$

$s_{j, x}^{(\text {eff })} \equiv \sum_{n=1}^{N_{c}} A_{n}\left(M_{x} M_{x+\hat{j}}\right)^{n}+A_{j, x}\left(\bar{B}_{x} B_{x+\hat{j}}+(-1)^{N_{c}}(\right.$ H.c. $\left.)\right)$

where $B_{x}=\epsilon^{a b \cdots c}\left(\chi^{a} \chi^{b} \cdots \chi^{c}\right)_{x} / N_{c}$ ! represents baryonic composites. Coefficients $\left(A_{n}, A_{j, x}\right)$ are summarized in Table I for $N_{c}=3$. The spatial partition function in the SCL is obtained as

$$
Z_{\mathrm{SCL}}^{(s)}=\exp \left[-\sum_{j, x} s_{j, x}^{(\mathrm{eff})}\right]
$$

The sum over spatial directions $\sum_{j}$ in Eq. (13) would give rise to a factor $d$ due to the spatial isotropy. Suppose that the action $\sum_{j, x} s_{j, x}^{(\text {eff })}$ stays finite at large $d$, the quark field $(\chi, \bar{\chi})$ should scale as $d^{-1 / 4}$. As a result, the mesonic hopping term $\sum_{j} M_{x} M_{x+\hat{j}}$ remains finite $\mathcal{O}\left(1 / d^{0}\right)$, while higher power terms of quarks are found to be suppressed as $\mathcal{O}(1 / \sqrt{d})$ for $N_{c} \geq 3$. This is called the systematic $1 / d$ expansion, which is proposed first in the application to the Ising model [78]. A spin exchanging term $\sum_{j} S_{x} S_{x+\hat{j}}$ is assumed to be finite at large $d$, and the mesonic hopping $\sum_{j} M_{x} M_{x+\hat{j}}$ could be analogue of that [30]. In the leading order of the $1 / d$ expansion, the SCL spatial partition function becomes

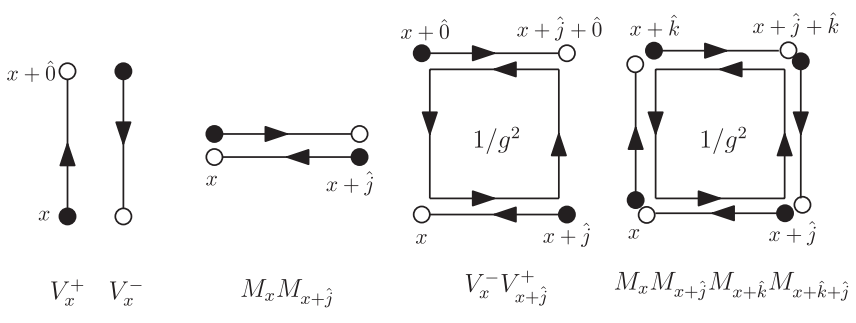

FIG. 1. Effective action terms in the strong coupling limit and $1 / g^{2}$ corrections. Open circles, filled circles, and arrows show $\chi$, $\bar{\chi}$, and $U_{\nu}$, respectively.
PHYSICAL REVIEW D 80, 074034 (2009)

$$
Z_{\mathrm{SCL}}^{(s)}=\exp \left[\frac{1}{4 N_{c}} \sum_{j, x} M_{x} M_{x+\hat{j}}+\mathcal{O}(1 / \sqrt{d})\right] .
$$

In the third diagram of Fig. 1, we display the leading order diagram of the $1 / d$ expansion.

\section{Strong coupling and cluster expansion}

In order to evaluate the plaquette contribution $S_{G}$, it is useful to define an expectation value,

$$
\langle\mathcal{O}\rangle=\frac{1}{Z_{\mathrm{SCL}}^{(s)}} \int \mathcal{D} U_{j} \mathcal{O}\left[U_{j}\right] e^{-S_{F}^{(s)}},
$$

which has a normalization property $\langle\mathbf{1}\rangle=1$. The full spatial partition function Eq. (7) can be expressed as

$$
Z^{(s)}=Z_{\mathrm{SCL}}^{(s)}\left\langle e^{-S_{G}}\right\rangle .
$$

It is well known that the expectation value of the exponential form operator with a small factor (i.e. $1 / g^{2}$ ) can be evaluated by using the cumulant expansion [79],

$$
\left\langle e^{-S_{G}}\right\rangle=\sum_{n=0}^{\infty} \frac{(-1)^{n}}{n !}\left\langle S_{G}^{n}\right\rangle=\exp \left[\sum_{n=1}^{\infty} \frac{(-1)^{n}}{n !}\left\langle S_{G}^{n}\right\rangle_{c}\right]
$$

where $\langle\cdots\rangle_{c}$ is called a cumulant, and corresponds to the correlation part in the connected diagram contributions, e.g. $\left\langle S_{G}^{2}\right\rangle_{c}=\left\langle S_{G}^{2}\right\rangle-\left\langle S_{G}\right\rangle^{2}$. We find that the effective action from plaquettes is expressed in terms of cumulants as

$$
\Delta S_{\text {eff }} \equiv-\log \left\langle e^{-S_{G}}\right\rangle=-\sum_{n=1}^{\infty} \frac{(-1)^{n}}{n !}\left\langle S_{G}^{n}\right\rangle_{c} .
$$

The $n$-th term in the right-hand side is proportional to $1 / g^{2 n}$, and we can identify $n=1$ term as the NLO effective action, and $n=2$ term as the next-to-next-to-leading order (NNLO) effective action.

The above identification of the effective action and the strong coupling order is consistent with the cluster expansion. In the first line of Eq. (17), the average of $S_{G}^{n}$ is decomposed into cumulants as

$$
\begin{aligned}
\left\langle S_{G}^{n}\right\rangle & =\sum_{\text {partition }} \prod_{\alpha}\left\langle S_{G}^{n_{\alpha}}\right\rangle_{c} \\
& =\left\langle S_{G}\right\rangle_{c}^{n}+\frac{n !}{2 !(n-2) !}\left\langle S_{G}\right\rangle_{c}^{n-2}\left\langle S_{G}^{2}\right\rangle_{c}+\cdots+\left\langle S_{G}^{n}\right\rangle_{c},
\end{aligned}
$$

where the sum runs over all partitions satisfying $\sum_{\alpha} n_{\alpha}=$ $n$. The plaquette action $S_{G}$ is proportional to a large volume factor $\sum_{x} \sim$ Vol and a small coupling factor $1 / g^{2}$, hence it is necessary to count both of them. The first term in Eq. (19) is estimated as

$$
\left\langle S_{G}\right\rangle_{c}^{n} \propto\left[\frac{1}{g^{2}} \sum_{x}\right]^{n} \sim \mathcal{O}\left(1 / g^{2 n}, \mathrm{Vol}^{n}\right) .
$$

In comparison, other terms have smaller powers in volume. 
For example, the cumulant of the $n$-th power operator is proportional to $(\mathrm{Vol})^{1}$,

$$
\left\langle S_{G}^{n}\right\rangle_{c} \propto \sum_{\left\{x_{i}\right\} \in \mathrm{conn}}\left[\frac{1}{g^{2 n}}\right] \sim \mathcal{O}\left(1 / g^{2 n}, \operatorname{Vol}^{1}\right),
$$

where " $\left\{x_{i}\right\} \in$ conn" represents that the sum over $\left\{x_{i} \mid i=\right.$ $1, \cdots, n\}$ is restricted to connected diagrams, and such a summation is $\mathcal{O}\left(\mathrm{Vol}^{1}\right)$. In a fixed order of $1 / g^{2 n},\left\langle S_{G}\right\rangle_{c}^{n}$ gives the leading order contribution in terms of the volume in the thermodynamical limit, $\mathrm{Vol} \rightarrow \infty$. Resumming all leading order contributions in volume of the connected diagrams, we obtain the exponential form shown in the right-hand side of Eq. (18). This resummation corresponds to the so-called cluster expansion [79], and is consistent with the strong coupling expansion of the effective action presented in Eq. (18).

\section{NLO effective action}

The NLO contribution to the effective action Eq. (18) is found to be

$$
\Delta S_{\mathrm{NLO}}=\left\langle S_{G}\right\rangle_{c}=-\frac{1}{g^{2}} \sum_{P}\left\langle U_{P}+U_{P}^{\dagger}\right\rangle_{c} .
$$

As long as we consider NLO terms, the cumulant $\langle\cdots\rangle_{c}$ is equivalent to the expectation value $\langle\cdots\rangle$,

$$
\left\langle U_{P}\right\rangle_{c}=\left\langle U_{P}\right\rangle=\left(1 / Z_{\mathrm{SCL}}^{(s)}\right) \int \mathcal{D} U_{j} U_{P} e^{-S_{F}^{(s)}} .
$$

We decompose the spatial kinetic action $S_{F}^{(s)}$ to the plaquette related and nonrelated part,

$$
\begin{gathered}
S_{F}^{(s)} \rightarrow s_{P}+\sum_{(j, x) \notin P} s_{j, x}, \\
s_{P}=\sum_{(j, x) \in P} s_{j, x} .
\end{gathered}
$$

In the above sum $\sum_{P}$, four links, $(j, x),(k, x+\hat{j}),(j, x+$ $\hat{k}),(k, x)$ are included in a spatial plaquette $P=(j k, x)$ as shown in the fifth diagram of Fig. 1. The link integral in Eq. (23) is also decomposed as follows:

$$
\begin{aligned}
\left\langle U_{P}\right\rangle_{c}= & {\left[\prod_{(j, x) \in P} e^{s_{j, x}^{\text {(eff) }}}\right]\left[\int d U_{P} U_{P} \exp \left[-s_{P}\right]\right] } \\
& \times \prod_{(k, y) \notin P}\left[e^{s_{k, y}^{\text {(eff) }}} \int d U_{k, y} \exp \left[-s_{k, y}\right]\right] .
\end{aligned}
$$

The second line shows the plaquette nonrelated part, and is found to be unity from the definition of $s_{j, x}^{\text {(eff) }}$ shown in Eq. (11). The prefactor in the first line corresponds to the normalization factor in the plaquette related part. This factor is also found to be unity in the leading order of the $1 / d$ expansion, since $s_{j, x}^{\text {(eff) }}$ contains four quarks, and is proportional to $d^{-1}$ at large $d$.
The $U_{P}$ integral part in Eq. (26) contains at most only four links, and we can perform the link integrals by using the group integral formulas Eq. (10) again. For a temporal plaquette, $P=(j 0, x)$, we find $[31,36]$

$$
\left\langle U_{j 0, x}\right\rangle_{c}=-\frac{1}{4 N_{c}^{2}} V_{x}^{-}(\mu) V_{x+\hat{j}}^{+}(\mu)+\mathcal{O}\left(d^{-3 / 2}\right),
$$

where the first term in Eq. (27) corresponds to the leading order in the $1 / d$ expansion and shown in the fourth diagram of Fig. 1. Note that the temporal link variable $U_{0}$ remains in the nonlocal color singlet composites $V^{ \pm}$, and will be integrated out later. For a spatial plaquette, $P=$ $(j k, x), U_{P}$ integral in Eq. (26) is found to be [31,36],

$$
\left\langle U_{j k, x}\right\rangle_{c}=\frac{1}{16 N_{c}^{4}} M_{x} M_{x+\hat{j}} M_{x+\hat{k}} M_{x+\hat{k}+\hat{j}}+\mathcal{O}\left(d^{-5 / 2}\right),
$$

where $M M M M$ term is the leading order contribution and is illustrated in the fifth diagram of Fig. 1.

Substituting Eqs. (27) and (28) in Eq. (22), we obtain the NLO effective action from plaquettes, $\Delta S_{\mathrm{NLO}}$. The plaquette sum $\sum_{P}$ in $\Delta S_{\mathrm{NLO}}$ leads to $\sum_{j} \sim d$ and $\sum_{j k} \sim d^{2}$ for temporal and spatial plaquettes, respectively. Since the quark fields $(\chi, \bar{\chi})$ scales as $d^{-1 / 4}$, the composites in Eq. (27) and (28) scale as $V^{-} V^{+} \sim d^{-1}$ and $M M M M \sim$ $d^{-2}$. Putting all together, $V^{-} V^{+}$and $M M M M$ give $\mathcal{O}\left(1 / d^{0}\right)$ contributions in $\Delta S_{\mathrm{NLO}}$.

In the following, we consider the leading (SCL) and the NLO in the strong coupling expansion, and the leading order of the $1 / d$ expansion. The effective action is found to be

$$
\begin{aligned}
S_{\mathrm{eff}}= & S_{\mathrm{SCL}}+\Delta S^{(\tau)}+\Delta S^{(s)}+\mathcal{O}\left(1 / \sqrt{d}, 1 / g^{4}\right) \\
S_{\mathrm{SCL}}= & \frac{1}{2} \sum_{x}\left[V_{x}^{+}(\mu)-V_{x}^{-}(\mu)\right]+m_{0} \sum_{x} M_{x} \\
& -\frac{1}{4 N_{c}} \sum_{x, j>0} M_{x} M_{x+\hat{j}} \\
\Delta S^{(\tau)}= & \frac{\beta_{\tau}}{4 d} \sum_{x, j>0}\left[V_{x}^{+}(\mu) V_{x+\hat{j}}^{-}(\mu)+V_{x}^{+}(\mu) V_{x-\hat{j}}^{-}(\mu)\right] \\
\Delta S^{(s)}= & \frac{-\beta_{s}}{d(d-1)} \sum_{x, 0<k<j} M_{x} M_{x+\hat{j}} M_{x+\hat{k}} M_{x+\hat{k}+\hat{j}} \\
& \beta_{\tau}=\frac{d}{N_{c}^{2} g^{2}}, \quad \beta_{s}=\frac{d(d-1)}{8 N_{c}^{4} g^{2}}
\end{aligned}
$$

where $\Delta S^{(\tau)}$ and $\Delta S^{(s)}$ come from the temporal and spatial plaquettes shown in Eq. (27) and (28) including their hermit conjugates, respectively. The considered contributions $S_{F}^{(\tau)}, M M$, and $\Delta S^{(\tau, s)}$ are summarized in Fig. 1 . 


\section{EFFECTIVE POTENTIAL}

The effective action derived in the previous section still contains quark fields $(\chi, \bar{\chi})$ and temporal link variables $U_{0}$. In this section, we obtain the effective potential $\mathcal{F}_{\text {eff }}$ by integrating out these variables in the mean field approximation,

$$
\begin{aligned}
\int \mathcal{D}\left[U_{0}, \chi, \bar{\chi}\right] e^{-S_{\text {eff }}} & =\int \mathcal{D}[\Phi] e^{-N_{\tau} L^{d} \mathcal{F}_{\text {eff }}[\Phi]} \\
& \left.\approx e^{-N_{\tau} L^{d} \mathcal{F}_{\text {eff }}[\Phi]}\right|_{\text {stationary }}
\end{aligned}
$$

In this step, several auxiliary fields $(\Phi)$ including the chiral condensate $\sigma$ are introduced on the same footing, and the NLO effects are self-consistently evaluated.

\section{A. Effective potential in the strong coupling limit}

Before discussing the NLO effects, we briefly summarize the procedure to obtain the effective potential in the SCL. The effective action $S_{\mathrm{SCL}}$ contains the chirally invariant four-fermion interaction term $M M$. We apply the so-called Hubbard-Stratonovich (HS) transformation. The four-fermion interaction term $M M$ is reduced to bilinear forms in $\chi$ and $\bar{\chi}$ by performing the Gaussian transformation with a auxiliary field $\sigma$,

$$
\begin{aligned}
\exp \left[\sum_{x, j>0} \frac{M_{x} M_{x+\hat{j}}}{4 N_{c}}\right] & =e^{b_{\sigma} \sum_{x y} M_{x} V_{x y} M_{y} / 2}=\int \mathcal{D} \sigma e^{-\left(b_{\sigma} / 2\right)} \sum_{x y}\left[(\sigma+M)_{x} V_{x y}(\sigma+M)_{y}-M_{x} V_{x y} M_{y}\right] \\
& =\int \mathcal{D} \sigma \exp \left[-\sum_{x y} b_{\sigma}\left(\frac{\sigma_{x} V_{x y} \sigma_{y}}{2}+\sigma_{x} V_{x y} M_{y}\right)\right] \approx \exp \left[-N_{\tau} L^{d} \frac{b_{\sigma}}{2} \sigma^{2}-b_{\sigma} \sigma \sum_{x} M_{x}\right] \equiv e^{-S_{\sigma}},
\end{aligned}
$$

where $b_{\sigma}=d / 2 N_{c}$, and the matrix $V_{x y}=\sum_{j}\left(\delta_{x+\hat{j}_{, y}}+\right.$ $\left.\delta_{x-\hat{j}, y}\right) / 2 d$ represents the meson hopping. In the last line, $\sigma$ is assumed to be a constant, which is determined by the stationary condition $\partial \mathcal{F}_{\text {eff }}^{\mathrm{SCL}} / \partial \sigma=0$. Under this condition, the auxiliary field $\sigma$ is found to be the chiral condensate $\sigma=-\sum_{x}\left\langle M_{x}\right\rangle /\left(N_{\tau} L^{d}\right)$. Thus the nonlinear term $M M$ is converted to the quark mass term $b_{\sigma} \sigma \bar{\chi} \chi$, where the finite chiral condensate $\sigma$ spontaneously breaks the chiral symmetry and generates the quark mass dynamically.

Now the total effective action reduces to a bilinear form of $(\chi, \bar{\chi})$,

$$
\begin{aligned}
S_{\mathrm{SCL}} & \simeq \sum_{x}\left[\frac{V_{x}^{+}(\mu)-V_{x}^{-}(\mu)}{2}+m_{q} M_{x}\right]+N_{\tau} L^{d} \frac{b_{\sigma}}{2} \sigma^{2} \\
& \simeq \sum_{x y} \bar{\chi}_{x} G_{x y}^{-1}\left(m_{q}, \mu\right)_{\chi_{y}}+N_{\tau} L^{d} \frac{b_{\sigma}}{2} \sigma^{2},
\end{aligned}
$$

where $m_{q}=m_{0}+b_{\sigma} \sigma$ represents the constituent quark mass, and the inverse propagator of quarks is given as

$$
\begin{aligned}
G_{x y}^{-1}\left(m_{q}, \mu\right)= & \frac{\delta_{\mathbf{x y}}}{2}\left(e^{\mu} U_{0, x} \delta_{x+\hat{0}, y}-e^{-\mu} U_{0, x}^{\dagger} \delta_{x-\hat{0}, y}\right) \\
& +m_{q} \delta_{x y} .
\end{aligned}
$$

We take account of finite $T$ effects by imposing periodic and antiperiodic boundary conditions on link variables and quarks, respectively. We take the static and diagonalized gauge (called the Polyakov gauge) for temporal link variables with respect for the periodicity [34],

$$
U_{0}(\tau, \mathbf{x})=\operatorname{diag}\left(e^{i \theta_{1}(\mathbf{x}) / N_{\tau}}, \cdots, e^{i \theta_{N_{c}}(\mathbf{x}) / N_{\tau}}\right) .
$$

The corresponding Haar measure is given in the form of the Van der Monde determinant,

$$
\int d U_{0} \equiv\left[\prod_{a=1}^{N_{c}} \int_{-\pi}^{\pi} \frac{d \theta_{a}}{2 \pi}\right] \prod_{a<b}\left|e^{i \theta_{a}}-e^{i \theta_{b}}\right|^{2} 2 \pi \delta\left(\sum_{a} \theta_{a}\right),
$$

where the delta function reflects the $S U\left(N_{c}\right)$ property, i.e. the baryonic effect in the temporal direction. Owing to the static property of the auxiliary field $\sigma$ and the temporal link variable in the Polyakov gauge, the partition function $Z_{q}=$ $\int_{\chi, \bar{\chi}, U_{0}} e^{-\bar{\chi} G^{-1} \chi}$ is completely factorized in terms of the frequency modes. Hence the quark path integral can be done in each mode independently, and leads to the simple product in the frequency $\left(\prod_{\omega}\right)$. By utilizing the Matsubara method (see, for example, the appendices in Refs. [40,44]), we obtain the partition function as

$$
\begin{aligned}
Z_{q}= & \prod_{\mathbf{x}}\left[\int d U_{0 \mathbf{x}} e^{E_{q} / T} \prod_{a=1}^{N_{c}}\left[1+e^{-\left(E_{q}-\mu\right) / T+i \theta_{\mathbf{x}}^{a}}\right]\right. \\
& \left.\times\left[1+e^{-\left(E_{q}+\mu\right) / T+i \theta_{\mathbf{x}}^{a}}\right]\right]
\end{aligned}
$$

where $N_{\tau}^{-1}$ is identified as temperature $T$, and $E\left(m_{q}(\sigma)\right)=$ $\sinh ^{-1}\left[m_{q}(\sigma)\right]$ corresponds to the quark excitation energy. Substituting Eq. (39) for Eq. (40), the remnant $U_{0}$ integral can be carried out in a straightforward manner [34] (the explicit procedure is summarized in the appendix in Ref. [43]). The resultant effective potential [34,36] is a function of the chiral condensate $\sigma$, temperature $T$, and quark chemical potential $\mu$,

$$
\mathcal{F}_{\text {eff }}^{\mathrm{SCL}}(\sigma ; T, \mu)=\frac{b_{\sigma}}{2} \sigma^{2}+\mathcal{V}_{q}\left(m_{q}(\sigma) ; T, \mu\right),
$$




$$
\begin{gathered}
\mathcal{V}_{q}\left(m_{q} ; T, \mu\right)=-T \log \left[X_{N_{c}}\left(m_{q}\right)+2 \cosh \left[\frac{N_{c} \mu}{T}\right]\right], \\
X_{N_{c}}\left(m_{q}\right)=\frac{\sinh \left[\left(N_{c}+1\right) E_{q}\left(m_{q}\right) / T\right]}{\sinh \left[E_{q}\left(m_{q}\right) / T\right]} .
\end{gathered}
$$

The same result is also obtained by another method [36], by utilizing recursion formulas. The phase diagram is obtained by performing the minimum search of the effective potential $\mathcal{F}_{\text {eff }}^{\mathrm{SCL}}$, and its structure has been investigated in Refs. [37-39,42-44].

\section{B. Extended Hubbard-Stratonovich transformation}

We shall now evaluate NLO correction terms $\Delta S^{(\tau, s)}$ in the effective action Eq. (29) in the mean field approximation. The temporal plaquette term $\Delta S^{(\tau)}$ is composed of the product of different composites $V_{x}^{+}$and $V_{x \pm \hat{j}}^{-}$. The standard HS transformation shown in Eq. (35) cannot be applied for such a term. Hence we apply here a recently developed method named extended Hubbard-Stratonovich (EHS) transformation $[45,46]$. Let us consider to evaluate a quantity $e^{\alpha A B}$, where $(A, B)$ and $\alpha$ represent arbitrary composite fields and a positive constant, respectively. We can represent $e^{\alpha A B}$ in the form of a Gaussian integral over two auxiliary fields $(\varphi, \phi)$,

$$
\begin{aligned}
e^{\alpha A B} & =\int d \varphi d \phi e^{-\alpha\left\{(\varphi-(A+B) / 2)^{2}+(\phi-i(A-B) / 2)^{2}\right\}+\alpha A B} \\
& =\int d \varphi d \phi e^{-\alpha\left\{\varphi^{2}-(A+B) \varphi+\phi^{2}-i(A-B) \phi\right\}} .
\end{aligned}
$$

The integral over the new fields $(\varphi, \phi)$ is approximated by the saddle point value, $\varphi=\langle A+B\rangle / 2$ and $\phi=$ $i\langle A-B\rangle / 2$. Specifically in the case where both $\langle A\rangle$ and $\langle B\rangle$ are real, which applies to the later discussion, the stationary value of $\phi$ becomes purely imaginary. Thus we replace $\phi \rightarrow i \omega$ and require the stationary condition for the real value of $\omega$,

$$
\left.e^{\alpha A B} \approx e^{-\alpha\left\{\varphi^{2}-(A+B) \varphi-\omega^{2}+(A-B) \omega\right\}}\right|_{\text {stationary }}
$$

In the case of $A=B$, Eq. (45) reduces to the standard HS transformation. We find that $e^{\alpha A B}$ is invariant under the scale transformation, $A \rightarrow \lambda A$ and $B \rightarrow \lambda^{-1} B$. In our previous work [44], a similar invariance exists but is broken after the saddle point approximation. As a result, a careful treatment is necessary in order to determine the explicit value of the parameter. In the present derivation, the scale invariance is kept in the right-hand side of Eq. (45), since the combinations $\varphi-\omega=\langle A\rangle$ and $\varphi+\omega=\langle B\rangle$ transform in the same way as $A$ and $B$, respectively. This means that the effective potential is independent of the choice of $\lambda$.

Now we apply EHS to NLO terms. For the spatial plaquette action terms, $\Delta S^{(s)}$, we substitute $\left(\beta_{s} / d(d-1)\right.$,

$$
\begin{aligned}
\left.M_{x} M_{x+\hat{j}}, M_{x+\hat{k}} M_{x+\hat{k}+\hat{j}}\right) \text { in Eq. (45), and obtain } \\
\begin{aligned}
\Delta S^{(s)} \approx & \frac{\beta_{s}}{d(d-1)} \sum_{x, 0<k<j}\left[\varphi_{s}^{2}-\omega_{s}^{2}-\left(\varphi_{s}-\omega_{s}\right) M_{x} M_{x+\hat{j}}\right. \\
& \left.-\left(\varphi_{s}+\omega_{s}\right) M_{x+\hat{k}} M_{x+\hat{k}+\hat{j}}\right] \\
\approx & N_{\tau} L^{d} \frac{1}{2} \beta_{s} \varphi_{s}^{2}-\frac{\beta_{s} \varphi_{s}}{d} \sum_{x, j>0} M_{x} M_{x+\hat{j}}
\end{aligned}
\end{aligned}
$$

In the last line, we have assumed that the auxiliary fields take constant and isotropic values. Under this constant auxiliary field assumption, $\omega_{s}$ effects disappear and the sum $\sum_{0<k<j}$ leads to a factor $d(d-1) / 2$ for the $\varphi_{s}^{2}$ term. As shown in the last line, the coupling terms of $\varphi_{S}$ and $M$ are rearranged to the same form as the meson hopping term in the SCL effective action by using the translational invariance. This $M_{x} M_{x+\hat{j}} \varphi_{s}$ term can be absorbed into the meson hopping effects $M_{x} M_{x+\hat{j}}$ in the SCL,

$$
\frac{b_{\sigma}}{2 d} \sum_{x} M_{x} M_{x+\hat{j}} \rightarrow \frac{b_{\sigma}+2 \beta_{s} \varphi_{s}}{2 d} \sum_{x} M_{x} M_{x+\hat{j}} .
$$

Thus the spatial NLO contributions lead to a shift of the coefficient for the meson hopping effects, which can be evaluated by introducing the chiral condensate $\sigma$ via Eq. (35). The coefficient modification is cared by replacing $S_{\sigma}$ in Eq. (35) with

$$
\tilde{S}_{\sigma}=N_{\tau} L^{d} \frac{\tilde{b}_{\sigma}}{2} \sigma^{2}+\tilde{b}_{\sigma} \sigma \sum_{x} M_{x}
$$

where $\tilde{b}_{\sigma}=d /\left(2 N_{c}\right)+2 \beta_{s} \varphi_{s}$. The constituent quark mass is found to be modified as

$$
m_{q}^{\prime}=m_{0}+\tilde{b}_{\sigma} \sigma
$$

For the temporal plaquette action $\Delta S^{(\tau)}$, we substitute $(\alpha, A, B)=\left(\beta_{\tau} / 4 d,-V_{x}^{+}(\mu), V_{x+\hat{j}}^{-}(\mu)\right)$, and obtain

$$
\begin{aligned}
\Delta S^{(\tau)} \approx & \frac{\beta_{\tau}}{4 d} \sum_{x, j>0}\left[\varphi_{\tau}^{2}+\left[V_{x}^{+}(\mu)-V_{x+\hat{j}}^{-}(\mu)\right] \varphi_{\tau}-\omega_{\tau}^{2}\right. \\
& \left.-\left[V_{x}^{+}(\mu)+V_{x+\hat{j}}^{-}(\mu)\right] \omega_{\tau}+(j \leftrightarrow-j)\right] \\
\approx & N_{\tau} L^{d} \frac{\beta_{\tau}}{2}\left(\varphi_{\tau}^{2}-\omega_{\tau}^{2}\right) \\
& +\frac{\beta_{\tau}}{2} \sum_{x}\left[\left(\varphi_{\tau}-\omega_{\tau}\right) V_{x}^{+}(\mu)-\left(\varphi_{\tau}+\omega_{\tau}\right) V_{x}^{-}(\mu)\right] .
\end{aligned}
$$

In the last line, we again assume that the auxiliary fields $\varphi_{\tau}$ and $\omega_{\tau}$ are constant and isotropic, then $\sum_{j}$ leads to a factor $d$. We combine $V^{ \pm}$terms in Eq. (50) with those in the SCL temporal action $S_{F}^{(\tau)}$, and the coefficients of $V^{ \pm}$are found to become $Z_{\mp} / 2$, where $Z_{ \pm}=1+\beta_{\tau}\left(\varphi_{\tau} \pm \omega_{\tau}\right)$. We rewrite these coefficients as $Z_{ \pm}=Z_{\chi} \exp ( \pm \delta \mu)$. Thus the SCL term $S_{F}^{(\tau)}$ is modified by the temporal NLO effects as 
TABLE II. The auxiliary fields and their stationary values. In the stationary value of $\varphi_{\tau}, \varphi_{0}=N_{c}-Z_{\chi} \tilde{m}_{q}+\beta_{\tau} \omega_{\tau}^{2}$.

\begin{tabular}{lcc}
\hline \hline Auxiliary Fields & Mean Fields & Stationary Values \\
\hline$\sigma$ & $\langle-M\rangle$ & $-\left(1 / Z_{\chi}\right)\left(\partial \mathcal{V}_{q} / \partial \tilde{m}_{q}\right)$ \\
$\varphi_{s}$ & $\langle M M\rangle$ & $\sigma^{2}$ \\
$\varphi_{\tau}$ & $-\left\langle\left(V^{+}-V^{-}\right) / 2\right\rangle$ & $2 \varphi_{0} /\left(1+\sqrt{1+4 \beta_{\tau} \varphi_{0}}\right)$ \\
$\omega_{\tau}$ & $-\left\langle\left(V^{+}+V^{-}\right) / 2\right\rangle$ & $-\partial V_{q} / \partial \tilde{\mu}=\rho_{q}$ \\
\hline \hline
\end{tabular}

$$
\begin{aligned}
\tilde{S}_{F}^{(\tau)} & =\frac{1}{2} \sum_{x}\left[Z_{-} V_{x}^{+}(\mu)-Z_{+} V_{x}^{+}(\mu)\right] \\
& =\frac{Z_{\chi}}{2} \sum_{x}\left(e^{\mu} e^{-\delta \mu} \bar{\chi}_{x} U_{0, x} \chi_{x+\hat{0}}-e^{-\mu} e^{\delta \mu}(\text { H.c. })\right) \\
& =\frac{Z_{\chi}}{2} \sum_{x}\left[V_{x}^{+}(\tilde{\mu})-V_{x}^{-}(\tilde{\mu})\right],
\end{aligned}
$$

where

$$
Z_{\chi}=\sqrt{Z_{+} Z_{-}}, \quad e^{\tilde{\mu}}=e^{\mu} e^{-\delta \mu}=e^{\mu} \sqrt{\frac{Z_{-}}{Z_{+}}} .
$$

In this way, temporal NLO contributions are expressed as the quark wave function renormalization factor $Z_{\chi}$ and the dynamical shift of chemical potential $\delta \mu$. In Table II, we summarize introduced auxiliary fields.

\section{Effective potential}

Now the effective action reduces to a bilinear form in terms of the quark fields $(\chi, \bar{\chi})$,

$$
\begin{aligned}
S_{\text {eff }}= & Z_{\chi} \sum_{x y} \bar{\chi}_{x} G_{x y}^{-1}\left(\tilde{m}_{q}, \tilde{\mu}\right) \chi_{y} \\
& +N_{\tau} L^{d}\left[\frac{\tilde{b}_{\sigma}}{2} \sigma^{2}+\frac{\beta_{s}}{2} \varphi_{s}^{2}+\frac{\beta_{\tau}}{2}\left(\varphi_{\tau}^{2}-\omega_{\tau}^{2}\right)\right],
\end{aligned}
$$

where $G_{x y}^{-1}\left(\tilde{m}_{q}, \tilde{\mu}\right)$ is given in Eq. (37) with modifications $\left(m_{q}, \mu\right) \rightarrow\left(\tilde{m}_{q}, \tilde{\mu}\right)$. We note that the constituent quark mass is modified again due to the quark wave function renormalization factor $Z_{\chi}$,

$$
\tilde{m}_{q}=\frac{m_{q}^{\prime}}{Z_{\chi}}=\frac{m_{0}+\tilde{b}_{\sigma} \sigma}{Z_{\chi}} .
$$

The remnant integrals $\int_{\chi, \bar{\chi}, U_{0}} e^{-Z_{\chi} \sum \bar{\chi} G\left(\tilde{m}_{q}, \tilde{\mu}\right) \chi}$ can be evaluated in the same manner as in the SCL. The effective potential (free energy density) is obtained as a function of the auxiliary fields $\Phi=\left(\sigma, \varphi_{\tau, s}, \omega_{\tau}\right)$, the temperature $T$, and the quark chemical potential $\mu$,

$$
\begin{gathered}
\mathcal{F}_{\text {eff }}(\Phi ; T, \mu)=\mathcal{F}_{\text {aux }}(\Phi)+\mathcal{V}_{q}\left(\tilde{m}_{q}(\Phi) ; T, \tilde{\mu}\right), \\
\mathcal{F}_{\text {aux }}(\Phi)=\frac{\tilde{b}_{\sigma} \sigma^{2}}{2}+\frac{\beta_{s} \varphi_{s}^{2}}{2}+\frac{\beta_{\tau}}{2}\left(\varphi_{\tau}^{2}-\omega_{\tau}^{2}\right)-N_{c} \log Z_{\chi}
\end{gathered}
$$

where $\mathcal{V}_{q}\left(\tilde{m}_{q}(\Phi) ; T, \tilde{\mu}\right)$ has the same functional form as that in the SCL Eq. (42) except for modifications $m_{q} \rightarrow$ $\tilde{m}_{q}(\Phi)$ and $\mu \rightarrow \tilde{\mu}$. The additional term $-N_{c} \log Z_{\chi}$, which has no counterpart in the SCL, appears from the quark wave function renormalization factor $Z_{\chi}$ through the fermion determinant contribution, $-\log \left[\operatorname{det}\left(Z_{\chi} G^{-1}\right)\right]$.

We have introduced four kinds of auxiliary fields $\Phi=$ $\left(\sigma, \varphi_{s}, \varphi_{\tau}, \omega_{\tau}\right)$, and they may contain some redundant degrees of freedom. We can reduce their degrees of freedom by considering stationary conditions shown in Eq. (45),

$$
\frac{\partial \mathcal{F}_{\mathrm{eff}}}{\partial \Phi}=\frac{\partial \mathcal{F}_{\mathrm{aux}}}{\partial \Phi}+\frac{\partial \mathcal{V}_{q}}{\partial \tilde{m}_{q}} \frac{\partial \tilde{m}_{q}}{\partial \Phi}+\frac{\partial \mathcal{V}_{q}}{\partial \tilde{\mu}} \frac{\partial \tilde{\mu}}{\partial \Phi}=0 .
$$

Note that $\mathcal{V}_{q}$ depends on the auxiliary fields via the two dynamical variables $\tilde{m}_{q}$ and $\tilde{\mu}$. Substituting $\sigma$ for $\Phi$ in Eq. (57), we obtain the relation

$$
\sigma=-\frac{1}{Z_{\chi}} \frac{\partial \mathcal{V}_{q}}{\partial \tilde{m}_{q}} .
$$

By utilizing this result, the stationary condition for $\varphi_{s}$ leads to $\varphi_{s}=\sigma^{2}$. Substituting $\varphi_{\tau}$ and $\omega_{\tau}$ for $\Phi$, we obtain a coupled equation for $\varphi_{\tau}$ and $\omega_{\tau}$, whose solution is found to be

$$
\begin{gathered}
\varphi_{\tau}=\frac{2 \varphi_{0}}{1+\sqrt{1+4 \beta_{\tau} \varphi_{0}}}, \\
\varphi_{0}=N_{c}-Z_{\chi} \tilde{m}_{q}+\beta_{\tau} \omega_{\tau}^{2}, \\
\omega_{\tau}=-\frac{\partial \mathcal{V}_{q}}{\partial \tilde{\mu}}=-\frac{\partial \mathcal{F}_{\mathrm{eff}}}{\partial \mu} .
\end{gathered}
$$

Equation (61) indicates the stationary value of $\omega_{\tau}$ is nothing but the quark number density $\rho_{q}$. The stationary conditions are summarized in Table II.

The auxiliary fields $\varphi_{\tau, s}$ are found to be explicit functions of $\sigma$ and $\omega_{\tau}$ via stationary conditions, while $\omega_{\tau}$ becomes a $(T, \mu)$ dependent implicit function, $\omega_{\tau}=$ $\rho_{q}\left(\sigma, \omega_{\tau} ; \mu, T\right)$. Hence we need a self-consistent treatment in the minimum search of $\mathcal{F}_{\text {eff }}\left(\sigma, \omega_{\tau}\right)$ in order to determine vacua. This is a consequence of the multiorder parameter $\left(\sigma, \omega_{\tau}\right)$ treatment, and a new feature compared with the previous works [36-39].

The auxiliary field $\omega_{\tau}$ may be interpreted as a repulsive vector field for quarks. In relativistic mean field (RMF) models of nuclei [80], the isoscalar-vector field $\omega$ contributes to the energy density as

$$
\varepsilon_{V}=-m_{\omega}^{2} \omega^{2} / 2+g_{\omega N} \rho_{B}\left(\tilde{\mu}_{B}\right) \omega+\ldots,
$$

where $\omega$ is the temporal component of the omega meson 
field $\omega^{\nu}$. The negative coefficient of $\omega^{2}$ results in the repulsive potential for nucleons, $+g_{\omega N} \omega$, and the coupling with the baryon density $\rho_{B}$ leads to the shift of $\mu_{B}$ as

$$
E+g_{\omega} \omega-\mu_{B}=E-\left(\mu_{B}-g_{\omega} \omega\right)=E-\tilde{\mu}_{B} .
$$

The saddle point constraint gives $\omega \propto \rho_{B}$. Most of these characters apply to the auxiliary field $\omega_{\tau}$ introduced in the present work. For example, the $\omega_{\tau}$ contribution to the effective action in Eq. (50) is rewritten as $-\beta_{\tau} \omega_{\tau}^{2} / 2+$ $\beta_{\tau} \omega_{\tau} \rho_{q}$, and the stationary condition is $\omega_{\tau}=\rho_{q}$. When we replace quarks with baryons and introduce an appropriate scaling factor for $\omega_{\tau}$, the above two points are consistent with the properties of $\omega$ vector field in the RMF.

\section{PHASE DIAGRAM EVOLUTION}

In the previous section, we have derived an analytic expression of the effective potential $\mathcal{F}_{\text {eff }}$, which contains effects of the NLO of the $1 / g^{2}$ expansion. In this section, we investigate the phase diagram evolution with the finite coupling effects $\beta=2 N_{c} / g^{2}$ based on the effective potential $\mathcal{F}_{\text {eff }}$. By developing a self-consistent treatment of two order parameters, $\left(\sigma, \omega_{\tau}\right)$, we study the $\beta$ dependence of the critical temperature, critical chemical potential, the critical point, and the phase diagram. We also discuss PCR matter.

\section{A. Self-consistent treatment in vacuum search}

Solving the stationary condition of $\mathcal{F}_{\text {eff }}$ with respect to $\sigma$ and $\omega_{\tau}$,

$$
\frac{\partial \mathcal{F}_{\text {eff }}}{\partial \sigma}=\frac{\partial \mathcal{F}_{\text {eff }}}{\partial \omega_{\tau}}=0
$$

corresponds to searching for a saddle point of $\mathcal{F}_{\text {eff }}$ in the $\left(\sigma, \omega_{\tau}\right)$ plane. Since the quark number density $\rho_{q}$ is an increasing function of $\tilde{\mu}$ which is a decreasing function of $\omega_{\tau}$, the stationary condition for $\omega_{\tau}, \omega_{\tau}=\rho_{q}$, has a single solution, $\omega_{\tau}=\omega_{\tau}{ }^{\text {stat }}(\sigma, T, \mu)$, for a given value of $\sigma$ at finite $T$. The coefficient of $\omega_{\tau}{ }^{2}$ is negative in $\mathcal{F}_{\text {eff }}$, hence the solution gives a maximum of $\mathcal{F}_{\text {eff }}$ for a given $\sigma$. Thus the stationary point in $\left(\sigma, \omega_{\tau}\right)$ is the saddle point of $\mathcal{F}_{\text {eff }}$, at which $\mathcal{F}_{\text {eff }}$ is convex downward and upward in $\sigma$ and $\omega_{\tau}$ directions $\left(\partial^{2} \mathcal{F}_{\text {eff }} / \partial \sigma^{2}>0\right.$ and $\left.\partial^{2} \mathcal{F}_{\text {eff }} / \partial \omega_{\tau}^{2}<0\right)$, respectively. Generally, we may have several solutions of Eq. (64), among which the lowest $\mathcal{F}_{\text {eff }}$ dominates the partition function.

In Fig. 2, we show $\mathcal{F}_{\text {eff }}$ as a function of $\left(\sigma, \omega_{\tau}\right)$. Solid lines show the solution of the stationary condition for $\omega_{\tau}$, $\omega_{\tau}=\omega_{\tau}{ }^{\text {stat }}$, and filled circles show the saddle points. At $\mu=0, \mathcal{F}_{\text {eff }}$ becomes an even function of $\omega_{\tau}$, and the stationary value of $\omega_{\tau}$ is always zero as shown in the upper panel of Fig. 2. At finite $\mu$, we have to solve the coupled Eqs. (64) self-consistently. The solution $\omega_{\tau}{ }^{\text {stat }}$ is a smooth function of $\sigma$ at finite $T$ as shown in the middle panel of
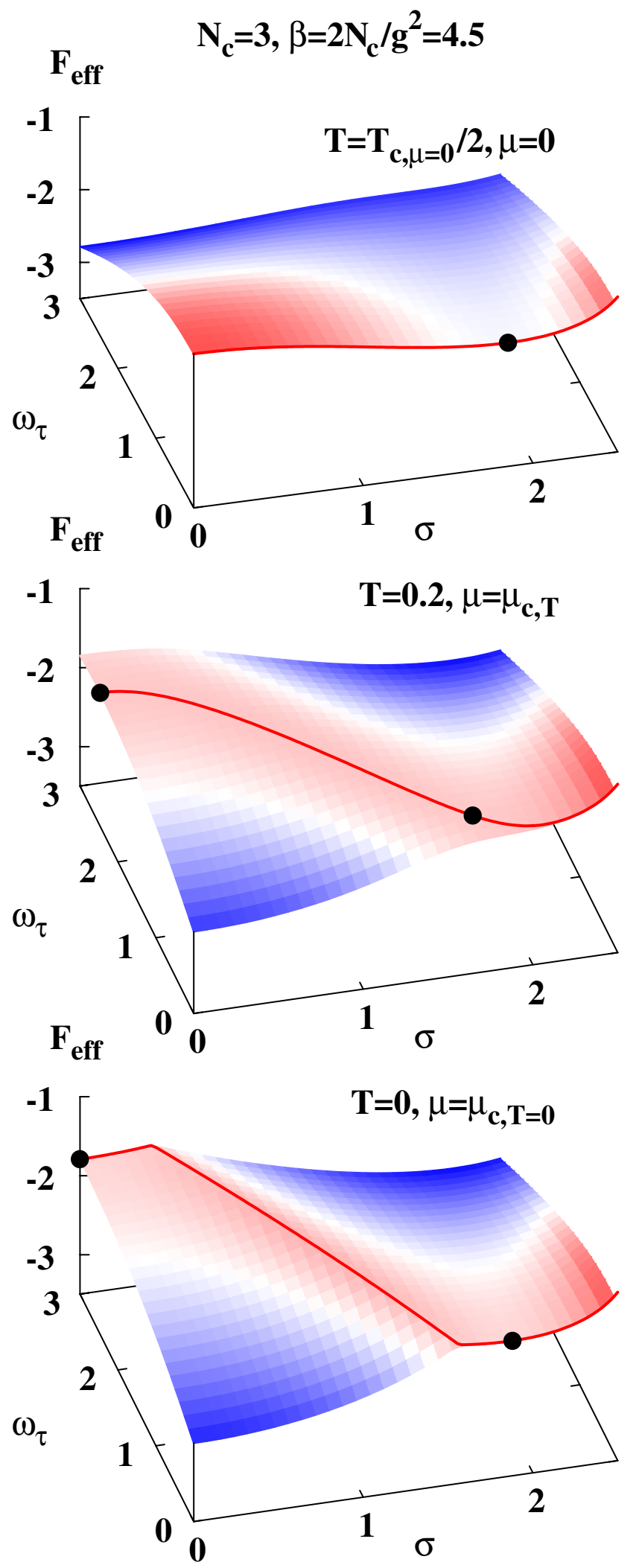

FIG. 2 (color online). The effective potential as a function of $\sigma$ and $\omega_{\tau}$ at $(T, \mu)=\left(T_{c, \mu=0} / 2,0\right),\left(0.2, \mu_{c, T=0.2}\right),\left(0, \mu_{c, T=0}\right)$ in the lattice unit. The solid line represents the set of points which satisfy the stationary condition Eq. (61). 
Fig. 2. In the case of $T=0$ and finite $\mu, \partial \mathcal{F}_{\text {eff }} / \partial \omega_{\tau}$ is discontinuous at $\tilde{\mu}=E_{q}$. This discontinuity comes from the functional form of the quark free energy at $T=0$,

$$
\mathcal{V}_{q}\left(\tilde{m}_{q}, \tilde{\mu}, T=0\right)= \begin{cases}-N_{c} E_{q} & \left(E_{q} \geq \tilde{\mu}\right) \\ -N_{c} \tilde{\mu} & \left(E_{q} \leq \tilde{\mu}\right)\end{cases}
$$

The ridge found in the lower panel of Fig. 2 corresponds to the line $\tilde{\mu}=E_{q}$, where $\mathcal{F}_{\text {eff }}$ takes a maximum value for a given $\sigma$. The stationary value $\omega_{\tau}^{\text {stat }}$ at finite $T$ approaches this ridge in the limit $T \rightarrow 0$. Thus the stationary condition for $\omega_{\tau}$ is found to be equivalent to searching for $\omega_{\tau}$ which maximizes $\mathcal{F}_{\text {eff }}$ for each $\sigma$ also at $T=0$.

The effective potential as a function of $\sigma$ for given $(T, \mu)$ is defined as $\mathcal{F}_{\text {eff }}(\sigma)=\mathcal{F}_{\text {eff }}\left(\sigma, \omega=\omega^{\text {stat }}(\sigma)\right)$, whose minimum point corresponds to the equilibrium. In Fig. 3, we show $\mathcal{F}_{\text {eff }}(\sigma)$ on the $T$ axis $(\mu=0)$ and on the $\mu$ axis $(T=0)$ at $\beta=4.5$, as an example. The chiral phase transitions in these cases are found to be the second and first order, respectively, as in the case of the SCL. In the following subsections, we discuss the nature of these phase transitions.

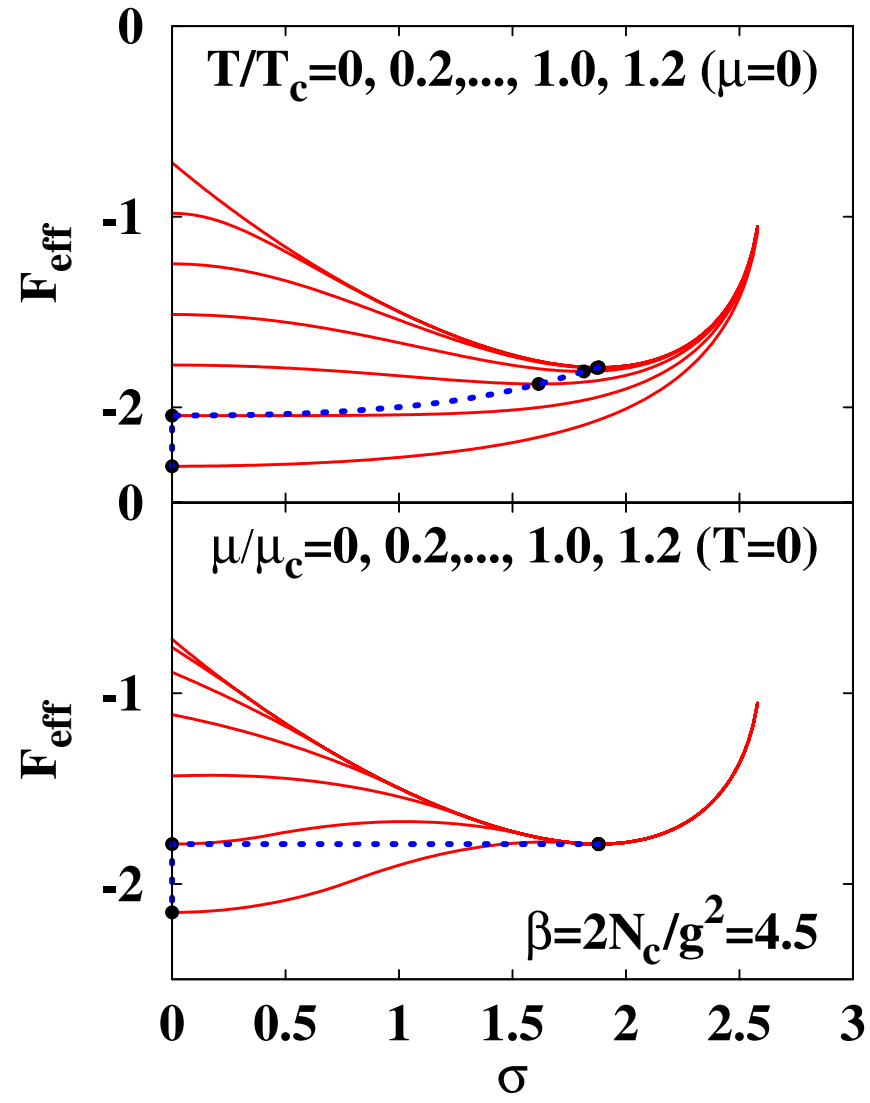

FIG. 3 (color online). The effective potential as a function of $\sigma$ on the $T$ axis (upper panel, $\mu=0$ ) and on the $\mu$ axis (lower panel, $T=0$ ) with $\beta=2 N_{c} / g^{2}=4.5$ in the lattice unit. The filled circles represent the minimum points.

\section{B. Critical temperature at zero chemical potential}

Along the $T$ axis $(\mu=0)$, the quark number density $\rho_{q}=\omega_{\tau}$ is always zero, then the effective potential under the $\omega_{\tau}$ stationary condition is simply given as $\mathcal{F}_{\text {eff }}(\sigma)=$ $\mathcal{F}_{\text {eff }}\left(\sigma, \omega_{\tau}=0\right)$. In the upper panel of Fig. 3, we show the effective potential at several temperatures $\left(T / T_{c}=\right.$ $0,0.2, \ldots, 1.0,1.2)$ at $\mu=0$ for $\beta=4.5$. The effective potential has one local minimum in the region $\sigma \geq 0$. As $T$ becomes large, the minimum point of the effective potential smoothly decreases to zero from a finite value. We find that the phase transition along the $T$ axis is the second order as in the case of the SCL [42-44].

In Fig. 4, we show the critical temperature at zero chemical potential $T_{c, \mu=0}$ as a function of $\beta$. We find that $T_{c}$ is suppressed as $\beta$ becomes large. This decrease would be a natural consequence of finite coupling, since hadrons are less bound than in the SCL. In the present treatment, the decrease of $T_{c}$ is caused by the wave function renormalization factor $Z_{\chi}$ in Eq. (54), which has a similar effect to the temporal lattice spacing modification. The second order phase transition temperature is obtained from the condition $C_{2}=0$, where $\mathcal{F}_{\text {eff }}=\sum_{n} C_{n} \sigma^{n} / n !$. The effective potential $\mathcal{F}_{\text {eff }}(\sigma)$ and auxiliary fields $\Psi=$ $\left(\varphi_{\tau, s}, \omega_{\tau}\right)$ are even functions of $\sigma$ in the chiral limit $\partial \Psi /\left.\partial \sigma\right|_{\sigma \rightarrow 0}=0$, and the first derivative of auxiliary fields are zero from the stationary conditions $\partial \mathcal{F}_{\text {eff }} / \partial \Psi=0$. By using these, we find $C_{2}$ is given as

$$
\begin{aligned}
C_{2} & \left.\equiv\left(\frac{\partial}{\partial \sigma}+\sum_{\Psi} \frac{\partial \Psi}{\partial \sigma} \frac{\partial}{\partial \Psi}\right)^{2} \mathcal{F}_{\text {eff }} \rightarrow \frac{\partial^{2} \mathcal{F}_{\text {eff }}}{\partial \sigma^{2}}\right|_{\sigma=0} \\
& =\left[\frac{\partial^{2} \mathcal{F}_{\mathrm{aux}}}{\partial \sigma^{2}}+\frac{b_{\sigma}^{2}}{Z_{\chi}^{2}} \frac{\partial^{2} \mathcal{V}_{q}}{\partial \tilde{m}_{q}^{2}}\right]_{\sigma=0} \\
& =b_{\sigma}-\frac{b_{\sigma}^{2}}{Z_{\chi}^{2}} \frac{N_{c}\left(N_{c}+1\right)\left(N_{c}+2\right)}{3 T\left(N_{c}+1+2 \cosh \left(N_{c} \tilde{\mu} / T\right)\right)} .
\end{aligned}
$$

From the condition $C_{2}=0$ at $\mu=0$, we find

$$
T_{c, \mu=0}=\frac{T_{c}^{(\mathrm{SCL})}}{Z_{\chi}^{2}}=\frac{1}{Z_{\chi}^{2}} \frac{d\left(N_{c}+1\right)\left(N_{c}+2\right)}{6\left(N_{c}+3\right)},
$$

where $T_{c}^{(\mathrm{SCL})}$ represents the second order phase transition temperature at $\mu=0$ in the strong coupling limit. It should be noted that at $\mu=0$ and $\sigma=0, Z_{\chi}$ does not depend on the auxiliary field. As shown in Eq. (67), the critical temperature decreases due to the wave function renormalization factor, $Z_{\chi} \geq 1$ at $\sigma=0$. This mainly originates from the suppression of the constituent quark mass $\tilde{m}_{q}=$ $m_{q}^{\prime} / Z_{\chi}$. In this way, the decrease of the critical temperature is understood as the $\tilde{m}_{q}$ modification effects caused by the plaquettes. The $T_{c}$ values here are consistent with those in Ref. [39].

In Fig. 4, we also show the results of the critical coupling in Monte Carlo simulations with $N_{\tau}=2$ [81], 4 [10,76,82], and $N_{\tau}=8$ [83] temporal lattice sizes. These results cor- 


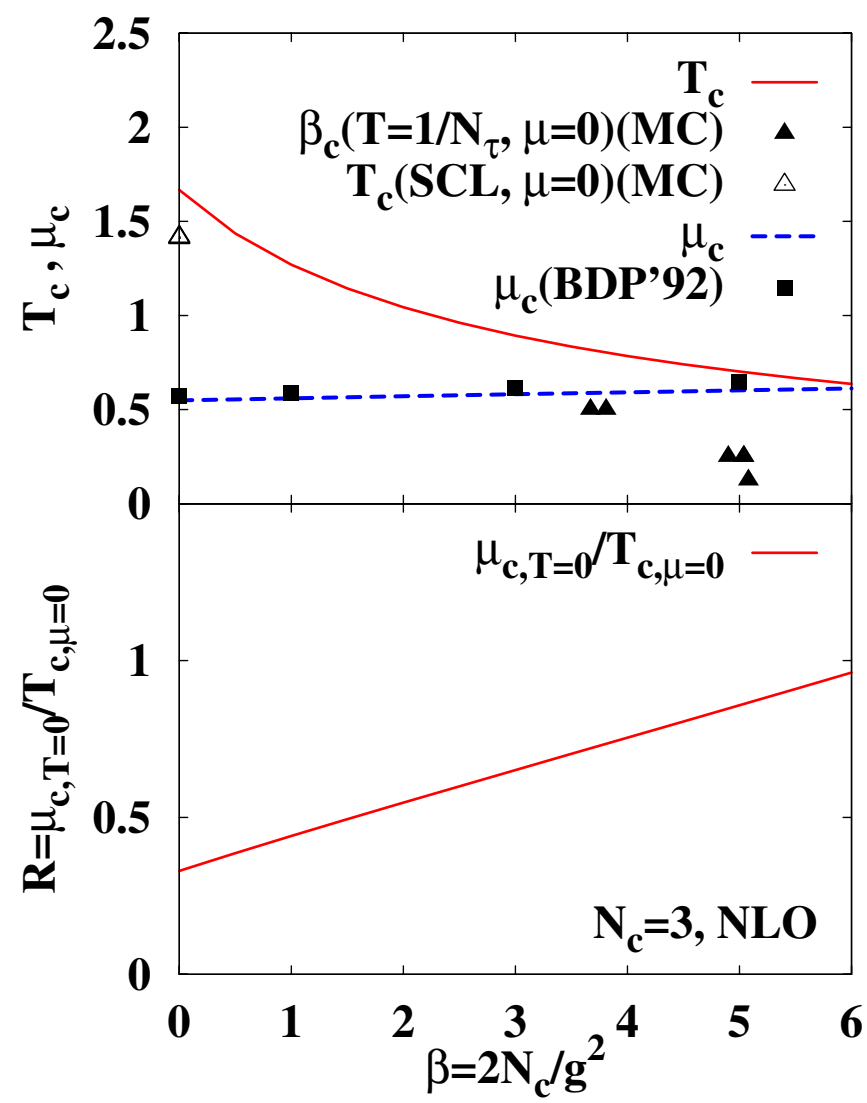

FIG. 4 (color online). Critical temperature, chemical potential, and critical coupling. In the upper panel, solid and dashed curves show the NLO results of $T_{c, \mu=0}$ and $\mu_{c, T=0}$, respectively. Solid squares show the results of $\mu_{c, T=0}$ in the previous work by Bilic, Demeterfi, and Petersson [37]. We also show the MC results of the critical coupling $\left(\beta_{c}\right)$ for given $N_{\tau}=1 / T=2,4$, and 8 at $\mu=0$ (filled triangles) and the critical temperature in the strong coupling limit (open triangles). From the left $[51,85]$ (the SCL result of $T_{c}$ with monomer-dimer-polymer simulations), [81] $\left(\beta_{c}=3.67(2), N_{\tau}=2\right.$, with a quark mass $\left.m_{0}=0.025\right)$, [81] $\left(\beta_{c}=3.81(2), N_{\tau}=2, m_{0}=0.05\right),[82]\left(\beta_{c}=4.90(3), 8^{3} \times 4\right.$ lattice, extrapolated to $\left.m_{0}=0\right)$, [10] $\left(\beta_{c}=5.037(3), 16^{3} \times 4\right.$ lattice, $\left.m_{0}=0.05\right)$, [76] $\left(\beta_{c}=5.040(2), 6^{3} \times 4\right.$ lattice, $m_{0}=$ $0.05)$, and [83] $\left(N_{\tau}=8\right.$, extrapolated to $\left.m_{0}=0\right)$. In the lower panel, we show the ratio $R=\mu_{c, T=0} / T_{c, \mu=0}$.

respond to $T_{c}=0.5,0.25$, and 0.125 . Results with $N_{\tau}=2$ and $N_{\tau}=4$ are those with $m_{0}=0.05,0.025$, or 0 (extrapolated), and chiral extrapolated results are shown for $N_{\tau}=$ 8. Compared with the results of the critical coupling $\beta_{c}=$ 5.097 in the quenched calculation with $N_{\tau}=2$ [84], $\beta_{c}$ is significantly smaller with finite masses, $\beta_{c}=3.81$ and 3.67 for $m_{0}=0.05$ and $m_{0}=0.025$ [81]. The monomerdimer-polymer simulations on anisotropic lattice $[51,85]$ give the critical temperature $T_{c}=\gamma_{c}^{2} / N_{\tau}=1.401$ (2) [51], where $\gamma_{c}$ is the critical anisotropy in the chiral limit. The decrease of $T_{c}$ in the NLO at finite $\beta$ is not enough to explain these $\mathrm{MC}$ results, and higher order effects such as the NNLO and Polyakov loop effects would be necessary.

\section{Chiral transition at finite density}

At finite $\mu$, the quark number density $\rho_{q}=\omega_{\tau}$ is generally finite and depends on $\sigma$. We search for $\omega_{\tau}$ which maximizes $\mathcal{F}_{\text {eff }}\left(\sigma, \omega_{\tau}\right)$ for a given $\sigma$, and we substitute the solution, $\omega_{\tau}=\omega_{\tau}^{\text {stat }}(\sigma)$, in the effective potential. In the lower panel of Fig. 3, we show the effective potential at several chemical potentials $\left(\mu / \mu_{c}=0,0.2, \ldots, 1.0,1.2\right)$ at $T=0$ for $\beta=4$.5. The effective potential has one local minimum for $\mu$ smaller than the second order critical chemical potential, $\mu<\mu_{c, T=0}^{(2 \mathrm{nd})}$, and two local minima appear in the larger $\mu$ region. For $\beta=4.5$, the vacuum jumps from the Nambu-Goldstone $(\mathrm{NG})$ phase $\left(\sigma \simeq \sigma_{\text {vac }}\right)$ to the Wigner phase $(\sigma=0)$ at critical chemical potential, $\mu=\mu_{c}$, and this transition is the first order.

The first order chiral transition at finite $\mu$ necessarily involves the density gap. In the case of $\beta=4.5$ and $T=0$ shown in the lower panel of Fig. 2, the effective potentials at two points in $\left(\sigma, \omega_{\tau}\right)$ plane become equal at $\mu=\mu_{c}$, and the first order phase transition takes place. In the Wigner phase, the quark mass is small (zero in the chiral limit), then the quark number density is high. At high densities, the chemical potential effects are reduced as $\mu \rightarrow \tilde{\mu}=\mu-\delta \mu$ as discussed in the previous section.

In the upper panel of Fig. 4, we show the critical chemical potential at $T=0, \mu_{c, T=0}$, as a function of $\beta$. In the region of $\beta<6$, the phase transition at $T=0$ is the first order, as in the case of SCL results. We find that the first order critical chemical potential $\mu_{c, T=0}^{(1 \mathrm{st})}$ is not largely modified from the strong coupling limit value $\mu_{c}^{(\mathrm{SCL}, 1 \mathrm{st})} \simeq$ 0.55 . For example, we find $\mu_{c}^{(1 \mathrm{st})} \simeq 0.58(0.60)$ at $\beta=$ 3.0(4.5). This small modification is understood as follows: In the low temperature region, the first order phase transition is described in terms of the competition between the quark chemical potential and the constituent quark mass. Since the temporal plaquette suppresses both, the relative relations between them are not largely changed. Hence $\mu_{c}^{(1 \mathrm{st})} \simeq \mu_{c}^{(\mathrm{SCL}, 1 \mathrm{st})}$ follows. Results by Bilic et al. [37-39] are also shown in Fig. 4. Our results are qualitatively consistent with their results.

We can now discuss the critical value ratio $R=$ $\mu_{c, T=0} / T_{c, \mu=0}$, which characterizes the shape of the phase diagram. In the lower panel of Fig. 4, we show this ratio as a function of $\beta$. As already discussed, $T_{c, \mu=0}$ rapidly decreases as $\beta$ increases, while the finite coupling effects give rise to only small modifications of $\mu_{c, T=0}$. As a result, the ratio $R$ significantly increases with $\beta$ as shown in the lower panel of Fig. 4. The ratio $R$ becomes close to 1 at $\beta=6$, and much larger than the SCL results $R_{\mathrm{SCL}} \sim$ $0.3-0.45$ [42-44]. The lattice MC results indicate that the critical end point may locate in the region $\mu / T>1.0$, which suggests $R>1.0$. Based on the recent MC results $\left(T_{c}=170-200 \mathrm{MeV}\right)$ and a naïve estimate $N_{c} \mu_{c} \gtrsim M_{N}$ ( $M_{N}$ is the nucleon mass), the expected ratio in the real world would be $R=1.5 \sim 3$. Thus the finite coupling 
effects are found to increase the ratio $R$ and make it closer to the empirical value.

\section{Partially chiral restored matter}

One of the characteristic features of the present treatment is that the second order critical chemical potential $\mu_{c}^{(2 n d)}$ is finite even at $T=0$. The second order critical chemical potential $\mu_{c}^{(2 n d)}$ for a given $T$ is obtained by solving the condition $C_{2}=0$ at finite chemical potential. By using Eq. (66), the shifted second order critical chemical potential is obtained as

$$
\tilde{\mu}_{c}^{(2 \mathrm{nd})}=\frac{T}{N_{c}} \operatorname{arccosh}\left[1+\frac{\left(N_{c}+3\right)\left(T_{c}^{(\mathrm{SCL})} / Z_{\chi}^{2}-T\right)}{2 T}\right] .
$$

In the right-hand side, $\sigma=0$ is assumed. This equation is an implicit equation, which should be solved with the condition $\omega_{\tau}=\rho_{q}$ simultaneously; $Z_{\chi}$ in the right-hand side is a function of $\omega_{\tau}$, which is a function of $\tilde{\mu}$. The second order critical chemical potential differs from $\tilde{\mu}_{c}^{(2 \mathrm{nd})}$ by $\delta \mu$,

$$
\mu_{c}^{(2 \mathrm{nd})}=\tilde{\mu}_{c}^{(2 \mathrm{nd})}+\delta \mu=\tilde{\mu}_{c}^{(2 \mathrm{nd})}+\log \sqrt{Z_{+} / Z_{-}} .
$$

At $T=0, \tilde{\mu}_{c}^{(2 n d)}$ becomes zero. In the SCL, we do not have the second term in Eq. (69) and $\mu_{c}^{(2 n d)}$ approaches zero at small $T$. In the NLO, the second term in Eq. (69) is finite at finite $\beta$. As a result, there is a possibility that $\mu_{c}^{(2 n d)}$ overtakes $\mu_{c}^{(1 \mathrm{st})}$, which leads to the appearance of the partially chiral restored matter $[45,46]$.

We numerically find that $\mu_{c}^{(2 \mathrm{nd})}$ can overtake $\mu_{c}^{(1 \mathrm{st})}$ in the case $\beta \geqslant 4.5$. We show the phase diagrams with $\beta=3.0$, 4.5 , and 6.0 in comparison with the SCL phase diagram in Fig. 5. The TCP starts to deviate from the second order phase transition boundary at $\beta \sim 4.5$, and becomes a CEP at larger $\beta$ even in the chiral limit. When the CEP exists off the second order phase transition boundary, we have the temperature region, where the second order critical chemical potential is larger than the first order one, $\mu_{c}^{(2 n d)}(T)>$ $\mu_{c}^{(1 \mathrm{st})}(T)$. In this temperature region, $\mathcal{F}_{\text {eff }}$ at $\mu=\mu_{c}^{(1 \mathrm{st})}$ should have two local minima in the $\sigma>0$ region as shown in Fig. 6: At $\mu=\mu_{c}^{(1 \mathrm{st})}, \mathcal{F}_{\text {eff }}$ at two local minima are equal, and the local minimum with smaller $\sigma$ cannot be at $\sigma=0$, since the curvature of $\mathcal{F}_{\text {eff }}$ is negative at around $\sigma=0$ (i.e. $C_{2}<0$ ) in the chemical potential region $\mu<$ $\mu_{c}^{(2 \mathrm{nd})}$.

In the temperature region where the condition $\mu_{c}^{(2 \mathrm{nd})}>$ $\mu_{c}^{(1 \mathrm{st})}$ is satisfied, we have three chemical potential regions, $\mu<\mu_{c}^{(1 \mathrm{st})}, \mu_{c}^{(1 \mathrm{st})}<\mu<\mu_{c}^{(2 \mathrm{nd})}$, and $\mu>\mu_{c}^{(2 \mathrm{nd})}$. The vacuum is in the NG phase in the first region, where the chiral condensate is large enough. In the third region, the chiral condensate is completely zero, and it is in the Wigner

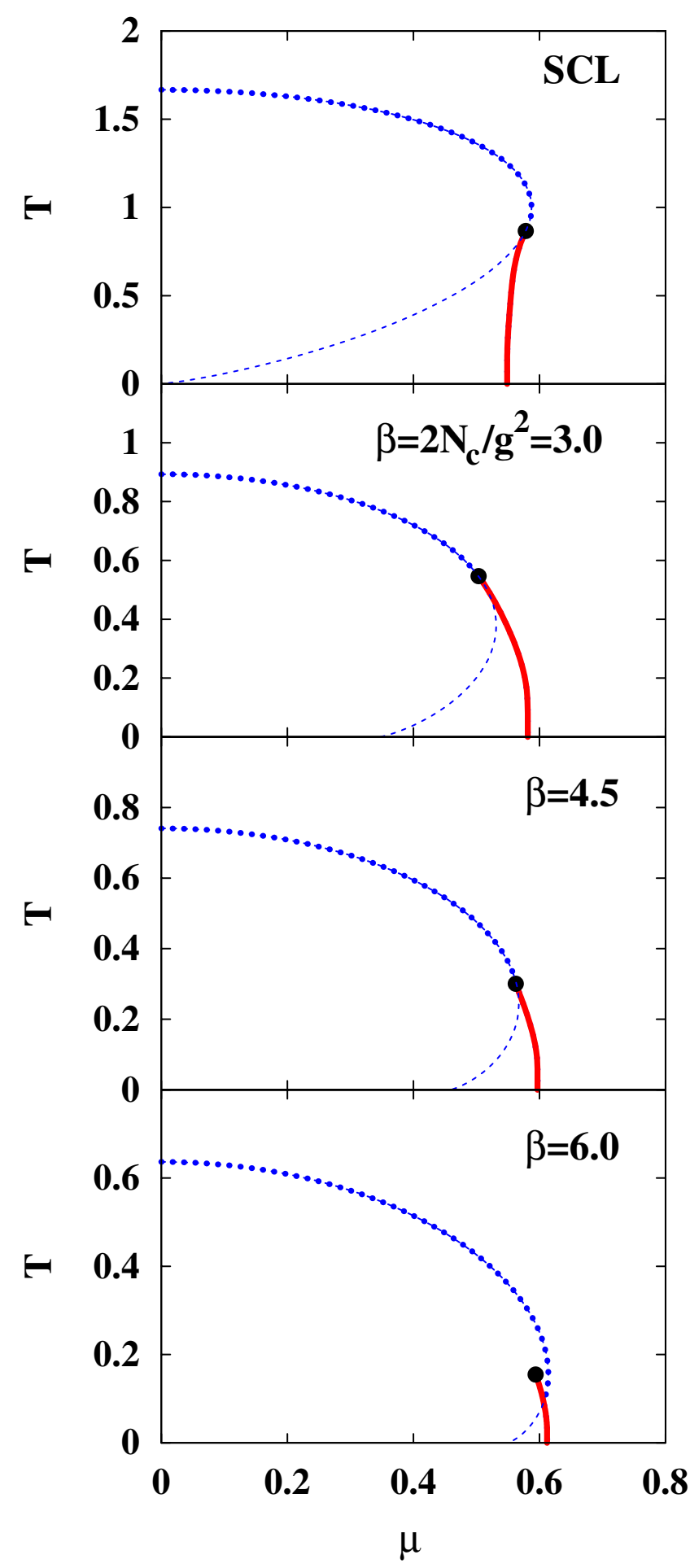

FIG. 5 (color online). The phase diagram for the several value of $\beta=2 N_{c} / g^{2}$ in the lattice unit. The solid and dashed lines represent the first and second order transition lines, respectively. The actual transition is described by the thick dashed and solid lines.

phase. In the second region, the chiral symmetry is weakly but spontaneously broken, and a PCR matter is realized $[45,46]$. It is interesting to investigate the transitions 


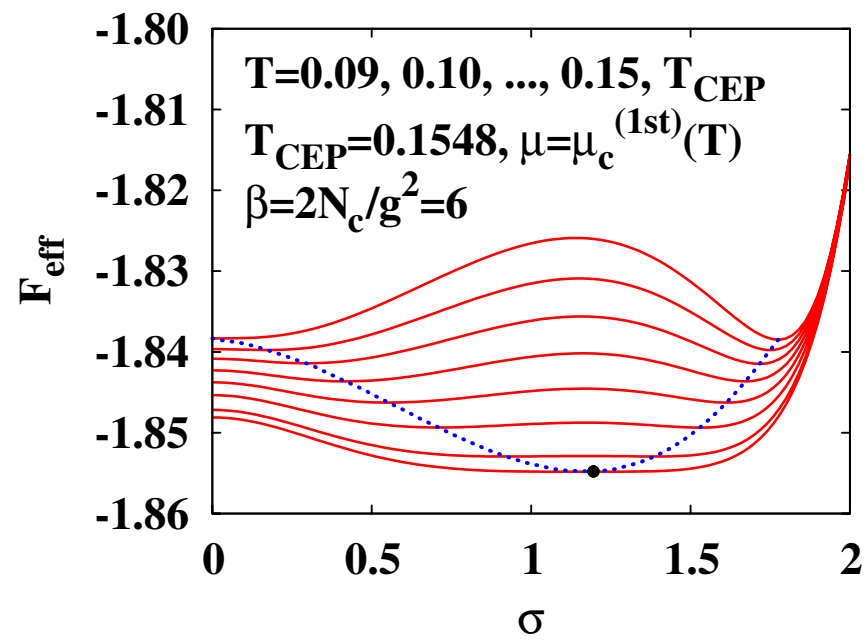

FIG. 6 (color online). Effective potential on the first phase transition boundary at $\beta=6$. Solid lines show the results at $T=$ $0.12,0.13,0.14,0.15$, and $T_{\mathrm{CEP}}$, where $T_{\mathrm{CEP}}=0.1548$, and dashed lines connect the coexisting equilibrium points on the boundary.

among them. As $\mu$ increases, the $\sigma$ jumps from the NG local minimum to the PCR local minimum with $\sigma>0$, as we can guess from the $\mathcal{F}_{\text {eff }}$ behavior in Fig. 6. For larger $\mu$, the chiral condensate in PCR matter decreases, and the Wigner phase $(\sigma=0)$ is realized at $\mu=\mu_{c}^{(2 \mathrm{nd})}$.

The appearance of PCR matter, or equivalently, $\mu_{c}^{(1 \mathrm{st})}<$ $\mu<\mu_{c}^{(2 \mathrm{nd})}$ region may stem from the multiorder parameter treatment $[45,46]$. To clarify this point, we examine several truncation schemes, where the effective potential $\mathcal{F}_{\text {eff }}\left(\sigma, \rho_{q}\right)$ systematically reduces to that with a single order parameter $\sigma$. And we would check the disappearance of the PCR matter.

The first treatment is the same as that we have discussed in previous subsections, and abbreviated as NLO-A. In the second treatment (NLO-B), $\mathcal{O}\left(1 / g^{4}\right)$ contributions in $Z_{\chi}$ and $\tilde{\mu}$ are truncated as

$$
\begin{aligned}
& Z_{\chi}^{(\mathrm{NLO}-\mathrm{B})}=1+\beta_{\tau} \varphi_{\tau}, \\
& \tilde{\mu}^{(\mathrm{NLO}-\mathrm{B})}=\mu-\beta_{\tau} \omega_{\tau} .
\end{aligned}
$$

In this treatment, we find that $\varphi_{\tau}$ and $\omega_{\tau}$ couple to quarks separately through $\tilde{m}_{q}$ and $\tilde{\mu}$, respectively. In the third prescription (NLO-C), we further truncate $\mathcal{O}\left(1 / g^{4}\right)$ terms in $\tilde{m}_{q}$ and in $\log Z_{\chi}$.

$$
\begin{gathered}
\tilde{m}_{q}^{(\mathrm{NLO}-\mathrm{C})}=\left(b_{\sigma} \sigma+m_{0}\right)\left(1-\beta_{\tau} \varphi_{\tau}\right)+2 \beta_{s} \varphi_{s} \sigma, \\
\Delta \mathcal{F}_{\mathrm{aux}} \equiv-N_{c} \log Z_{\chi} \approx-N_{c} \beta_{\tau} \varphi_{\tau} \quad \text { (NLO-C). }
\end{gathered}
$$

It is also possible to expand $V_{q}$ with respect to $\delta \mu=$ $\mu-\tilde{\mu}$ (NLO-D),

$$
V_{q}^{(\mathrm{NLO}-\mathrm{D})}\left(\tilde{m}_{q} ; \tilde{\mu}, T\right) \simeq V_{q}\left(\tilde{m}_{q} ; \mu, T\right)-\beta_{\tau} \omega_{\tau} \frac{\partial V_{q}}{\partial \mu}
$$

These truncation schemes are summarized in Table III.

Stationary conditions in NLO-B, C, and D are solved in a similar way to the NLO(NLO-A). In NLO-B and C, $\omega_{\tau}$ is still an implicit function of $\sigma, \omega_{\tau}=\rho_{q}\left(\sigma, \omega_{\tau} ; \mu, T\right)$, and the multiorder parameter property is still kept. In NLO-D, $\omega_{\tau}$ is explicitly obtained as a function of $\sigma$,

$$
\omega_{\tau}^{(\mathrm{NLO}-\mathrm{D})}=-\frac{\partial V_{q}\left(m_{q}(\sigma) ; \mu, T\right)}{\partial \mu},
$$

where the right-hand side does not contain $\omega_{\tau}$. The dynamics is described by a single order parameter $\sigma$. In this meaning, the NLO-D gives a similar formulation to those in the previous works [37-39].

In the upper panel of Fig. 7, we show the phase diagrams in NLO-A, B, and $\mathrm{C}$ in the large $\mu$ region. In NLO-A and $\mathrm{B}$, the maximum temperature of the first order phase boundary decreases, and the critical point deviates from the second order phase transition boundary at $\beta \simeq 4.5$ and 3.0 in NLO-A and NLO-B, respectively. In NLO-C, the second order critical chemical potential $\mu_{c}^{(2 n d)}$ at $T=0$ overtakes the first order one at $\beta \simeq 3.5$. Between the first and second order phase boundaries, we find PCR matter. In NLO-D, PCR matter does not appear in any region of $(T, \mu, \beta)$.

In the middle and lower panels of Fig. 7, we show the comparison of $\rho_{q}$ and $\sigma$ in the present treatments NLO-A, $\mathrm{B}, \mathrm{C}$, and $\mathrm{D}[45,46]$. The gradual increase of the quark number density $\rho_{q}$ after the first order transition is a common feature of the multiorder parameter treatments $[45,46]$. This means that $\rho_{q}$ is high, but still smaller than the maximum density $N_{c}$ in PCR matter at small $T$. At low

TABLE III. The truncation schemes in NLO-A, B, C, and D. In NLO-C and D, $\mathcal{O}\left(1 / g^{4}\right)$ terms in $\tilde{m}_{q}$ are truncated to be $\tilde{m}_{q}^{(\mathrm{NLO}-\mathrm{C}, \mathrm{D})}=$ $\left(b_{\sigma} \sigma+m_{0}\right)\left(1-\beta_{\tau} \varphi_{\tau}\right)+2 \beta_{s} \varphi_{s} \sigma$.

\begin{tabular}{lcccc}
\hline \hline & $\delta \mu$ & $\tilde{m}_{q}$ & $\Delta \mathcal{F}_{\text {aux }}$ & $\mathcal{V}_{q}$ \\
\hline NLO-A & $\log \sqrt{\frac{Z_{+}}{Z_{-}}}$ & $\frac{m_{q}}{\sqrt{Z_{+} Z_{-}}}$ & $-N_{c} \log \sqrt{Z_{+} Z_{-}}$ & $\mathcal{V}_{q}\left(\tilde{m}_{q}, \tilde{\mu}, T\right)$ \\
NLO-B & $\beta_{\tau} \omega_{\tau}$ & $\frac{m_{q}}{1+\beta_{\tau} \varphi_{\tau}}$ & $-N_{c} \log \left(1+\beta_{\tau} \varphi_{\tau}\right)$ & $\mathcal{V}_{q}\left(\tilde{m}_{q}, \tilde{\mu}, T\right)$ \\
NLO-C & $\beta_{\tau} \omega_{\tau}$ & $\tilde{m}_{q}^{(\mathrm{NLO}-\mathrm{C})}$ & $-N_{c} \beta_{\tau} \varphi_{\tau}$ & $\mathcal{V}_{q}\left(\tilde{m}_{q}, \tilde{\mu}, T\right)$ \\
NLO-D & 0 & $\tilde{m}_{q}^{(\mathrm{NLO}-\mathrm{D})}$ & $-N_{c} \beta_{\tau} \varphi_{\tau}$ & $\mathcal{V}_{q}\left(\tilde{m}_{q}, \mu, T\right)-\beta_{\tau} \omega_{\tau} \frac{\partial \mathcal{V}_{q}}{\partial \mu}$ \\
\hline \hline
\end{tabular}




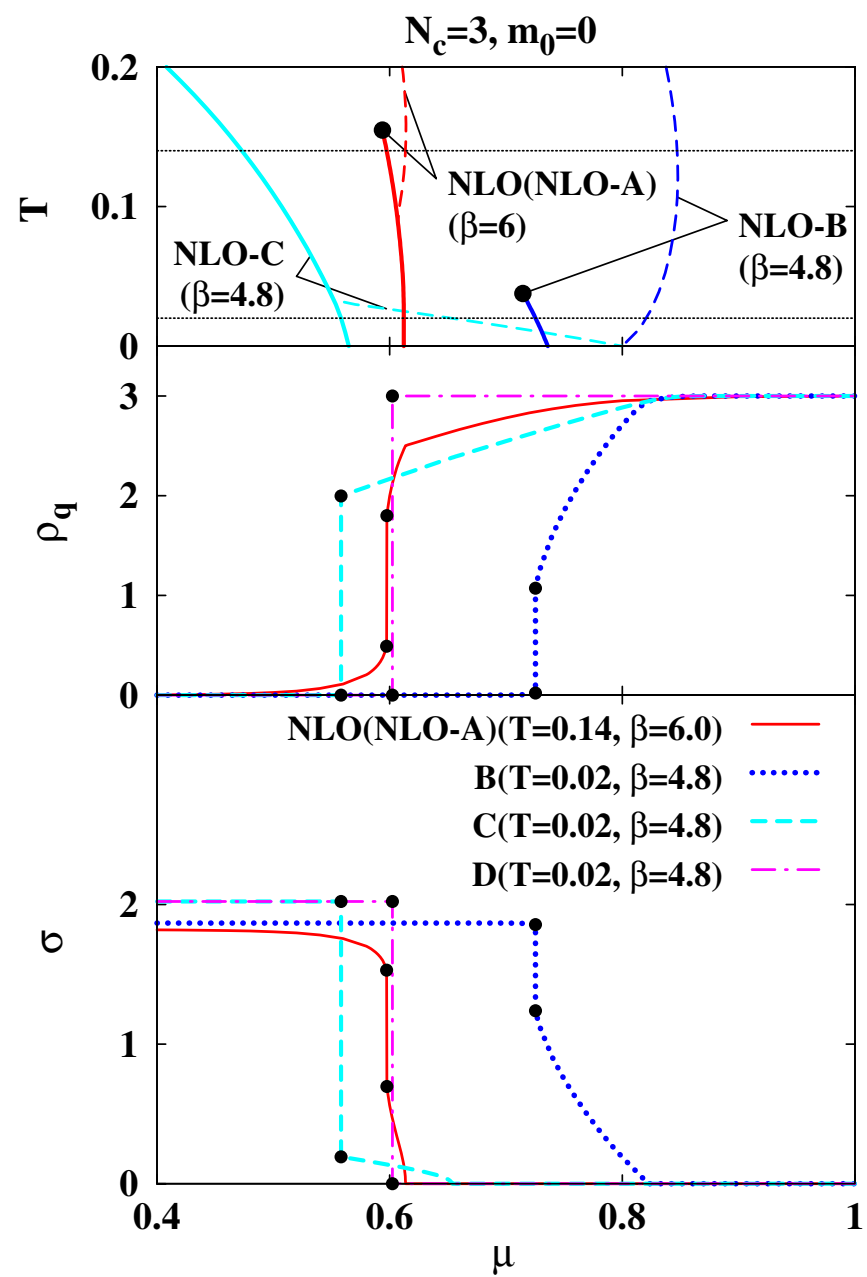

FIG. 7 (color online). In the upper panel, solid and dashed curves show the first and second order phase transition boundaries, and dots show the critical end point. In the middle and lower panels, solid, dotted, dashed, and dot-dashed curves show the results of NLO-A, B, C, and D, respectively, and dots and open squares show the first order transition points.

temperatures, we can investigate the appearance of the PCR matter more intuitively. The quark number density $\rho_{q}=-\partial \mathcal{F}_{\text {eff }} / \partial \mu$ is evaluated as

$$
\frac{\rho_{q}}{N_{c}}=\frac{2 \sinh \left[N_{c} \tilde{\mu} / T\right]}{X_{N_{c}}+2 \cosh \left[N_{c} \tilde{\mu} / T\right]} \underset{T \rightarrow 0}{\longrightarrow} \frac{x^{N_{c}}}{1+x^{N_{c}}},
$$

where $x=\exp \left[-\left(E_{q}-\tilde{\mu}\right) / T\right]$. When $E_{q}>\tilde{\mu}$ is satisfied at small $T$, we obtain $x \rightarrow 0$ and $\rho_{q} \rightarrow 0$, while $E_{q}<\tilde{\mu}$ leads to $x \rightarrow \infty$ and $\rho_{q} \rightarrow N_{c}$. Medium density $0<\rho_{q}<$ $N_{c}$ can appear only in the case where the energy and chemical potential balance, $E_{q}=\tilde{\mu}$, and $x$ stays finite at $T=0$. Since $\tilde{\mu}$ is a decreasing function of $\omega_{\tau}$, we may have a medium density solution of Eq. (76) in the region $\tilde{\mu}\left(\sigma, \omega_{\tau}=N_{c}\right)<E_{q}\left(\sigma, \omega_{\tau}\right)<\mu$. Specifically in NLO-B and $\mathrm{C}, E_{q}=\tilde{\mu}$ is found to be equivalent to the density condition $\rho_{q}=\left(\mu-E_{q}\right) / \beta_{\tau}$, which can take the a medium value. In the large $\beta$ region, this medium density matter can emerge in equilibrium and corresponds to the PCR matter as indicated in Fig. 7. Also in NLO-A, PCR matter appears in a similar mechanism at finite $T$. Thus the multiorder parameter treatment is essential to obtain the PCR matter at low $T$, and we observe the two chiral transitions as $\mu$ increases.

Now we have found following common properties as long as the quark number density is treated as the order parameter in addition to the chiral condensate $[45,46]$. (I) The PCR matter can appear in the large $\beta$ region; (II) The PCR matter sits next to the hadronic NG phase in the larger $\mu$ direction; (III) The quark number density is high as $\mathcal{O}\left(N_{c}\right)$ in PCR; (IV) In PCR matter, the effective chemical potential is adjusted to the quark excitation energy; and (V) The second order chiral transition to the Wigner phase follows after NG $\rightarrow$ PCR transition. All these properties would be the essence of the quarkyonic matter and transition proposed in Ref. [86]. In the previous work, the quark-driven Polyakov loop evaluated in SCLQCD is shown to be small as $\mathcal{O}\left(1 / N_{c}\right)$ [36], and it would not grow much at low temperatures. This feature is also consistent with the proposed property of the quarkyonic matter.

The quarkyonic matter is originally defined as the confined high density matter at large $N_{c}$ [86], and recently investigated by using the PNJL model [87]. In order to discuss the deconfinement dynamics, the higher order of $1 / g^{2}$ expansion would be essential, and a subject to be studied in future.

\section{E. Phase diagram evolution}

We shall now discuss the phase diagram evolution with $\beta$. In Fig. 8, we show dependence of the phase diagram on $\beta$ in the NLO(NLO-A). As $\beta$ increases, the second order phase boundary is compressed in the temporal direction according to the decrease of $T_{c}^{(2 n d)}$. The phase transition of cold $(T=0)$ dense matter is calculated to be the first order, and the critical chemical potential does not move much.

Thick lines in Fig. 8 show the coupling dependence of the first order phase transition boundaries. As is more clearly seen in Fig. 5, the slope of the first order phase transition boundary becomes negative at finite coupling, and it is natural from the Clausius-Clapeyron relation. We expect that the entropy and quark number density is higher in the Wigner phase, $\Delta s=s^{(\mathrm{W})}-s^{(\mathrm{NG})}>0$ and $\Delta \rho_{q}=$ $\rho_{q}^{(\mathrm{W})}-\rho_{q}^{(\mathrm{NG})}>0$, where $s^{(\mathrm{W}, \mathrm{NG})}$ and $\rho_{q}^{(\mathrm{W}, \mathrm{NG})}$ denote the entropy and quark number density, respectively, in the Wigner and the NG phases. With this expectation, the slope of the first order phase boundary from the ClausiusClapeyron relation, $d \mu / d T=-\Delta s / \Delta \rho_{q}$, should be negative. This improvement from the SCL may be related to the gradual increase of the quark number density in the PCR 


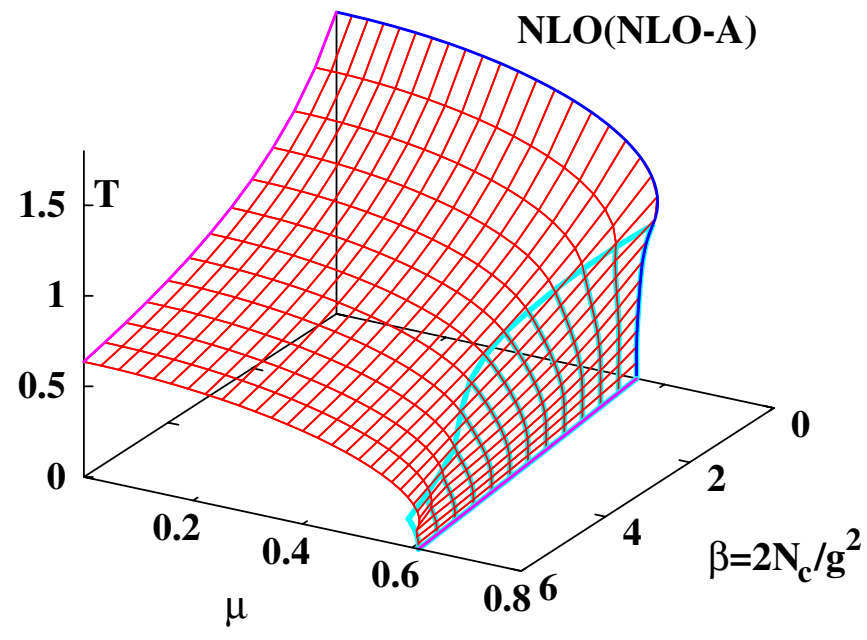

FIG. 8 (color online). The phase diagram evolution with the finite coupling effect $\beta=2 N_{c} / g^{2}$ in the lattice unit. Thin line surface shows the boundaries between the chiral broken and restored phases, and thick line surface shows the first order boundaries.

matter and in the Wigner phase. In the SCL, the quark number density jumps to an almost saturated value, $\rho_{q} \sim N_{c}$, at low $T$ at $\mu=\mu_{c}^{(1 \mathrm{st})}$. In this case, the lattice sites are almost filled by quarks, and the entropy density will be very small in the Wigner phase. This density saturation is a lattice artifact, and is expected to be weaken at finite $\beta$. With NLO effects, the vector field $\omega_{\tau}$ suppresses the sudden increase of $\rho_{q}$, and the quark number density gradually increases after the first order phase transition. We have discussed this feature in the PCR matter, and it also applies to the quark matter in the Wigner phase.

We also find that the slope $d \mu / d T$ is always negative in whole $T-\mu$ plane at finite $\beta$. This point is different from the previous NLO works [39].

The end point of the first order phase transition boundary is the critical point, which is either the TCP or the CEP. As we already discussed in the previous subsection, the TCP at small $\beta$ deviates from the second order phase transition line at $\beta \gtrsim 4.5$, and becomes the CEP. The temperature of this critical point gradually decreases, while the chemical potential stays in a narrow range as $\beta$ increases. We show the evolution of the critical point with $\beta$ in Fig. 9. The decrease of the critical point temperature, $T_{\mathrm{CP}}$, is consistent with the results in the NJL and PNJL models [88]. In these works, it is demonstrated that $T_{\mathrm{CP}}$ decreases as we adopt a larger vector coupling relative to the scalar coupling. In the present work, $\beta_{\tau} \omega_{\tau}$ is regarded as the vector potential for quarks, and it grows as $\beta$ increases.

In the continuum limit, one species staggered QCD would become the four flavor QCD with degenerate masses [75], where the chiral transition is expected to be the first order due to anomaly contributions [89]. The present behavior of the critical point shows that the NLO SC-LQCD does not contain anomaly effects.

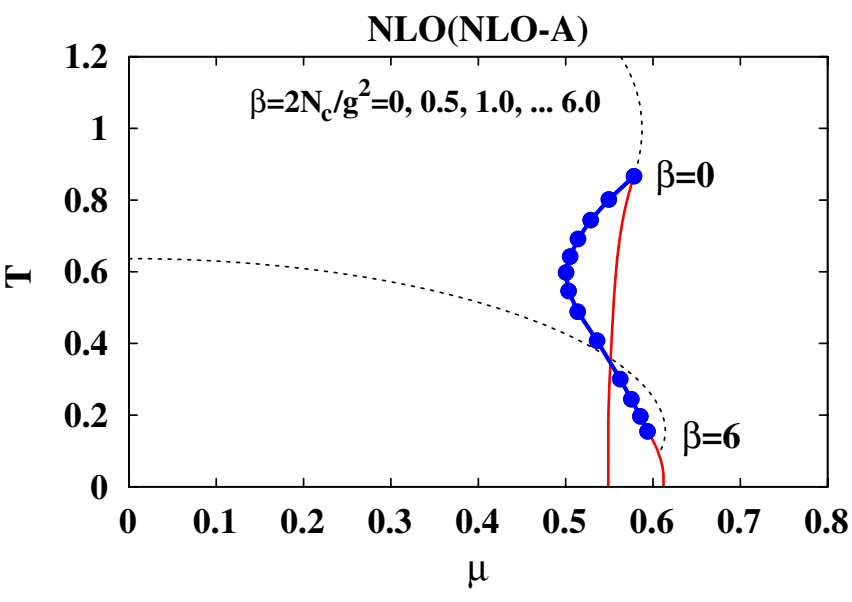

FIG. 9 (color online). Coupling dependence of the critical point. Filled circles show the critical points, and solid and dotted lines show the first and second order boundaries for $\beta=0$ and 6 .

\section{CONCLUDING REMARKS}

We have investigated the chiral phase transition in the strong coupling lattice QCD at finite temperature $(T)$ and chemical potential $(\mu)$ with finite coupling $\left(\beta=2 N_{c} / g^{2}\right)$ effects. We have derived an analytic expression of the effective potential using one species of staggered fermion in the leading (SCL) and NLO of the strong coupling $\left(1 / g^{2}\right)$ expansion and in the leading order of the large dimensional $(1 / d)$ expansion. We have focused our attention on the phase diagram evolution.

From the NLO effective action, we have derived the effective potential under the mean field approximation based on a self-consistent treatment of NLO effects with a recently proposed EHS transformation $[45,46]$. Then the quark number density $\left(\rho_{q}\right)$ is naturally introduced as an order parameter. NLO contributions are expressed via the shift of the constituent quark mass, dynamical chemical potential, and the quark wave function renormalization factor. The NLO effective potential is found to become a function of $T, \mu, \beta$, the chiral condensate $\sigma$, and quark number density $\rho_{q}$. Such a formulation has been essential in order to investigate the mechanism of the phase diagram evolution with $\beta$. The phase diagram has been obtained by performing the minimum search of the effective potential in the multiorder parameter treatment.

The effective constituent quark mass $\tilde{m}_{q}$ is found to be suppressed as $\beta$ increases. As a result, the critical temperature $T_{c}$ decreases and becomes closer to the Monte Carlo results at $\mu=0$ [81-83], while it is still larger than the MC data. The effective quark chemical potential $\tilde{\mu}$ is also suppressed as $\beta$ becomes larger. We have found the small modification in the critical chemical potential $\mu_{c}$ at low $T$. In this way, the ratio $R=$ $\mu_{c, T=0} / T_{c, \mu=0}$ becomes larger and closer to the empirical value. The $\beta$ dependences of $T_{c, \mu=0}$ and $\mu_{c, T=0}$ are consistent with the previous results [39]. The first order phase 
boundary is found to satisfy $d \mu / d T \leq 0$ at finite $\beta$. This behavior is natural from the Clausius-Clapeyron relation, and is different from the SCL results and previous results with NLO effects [39]. In the phase diagram evolution, the tricritical point is found to move in the lower $T$ direction. This trend is consistent with model results [88]. PCR matter is found to appear in the low $T$ and the large $\mu$ region with $\beta \gtrsim 4.5$. We have shown that the multiorder parameter $\left(\sigma, \omega_{\tau}\right)$ treatment is essential in describing PCR matter, where the effective chemical potential is automatically adjusted to the quark excitation energy.

We have discussed the NLO results in the region $\beta \leq 6$, expecting that the strong coupling expansion is convergent even in the region $\beta=5 \sim 6$. In the pure Yang-Mills theory, the character and strong coupling expansions seem to be convergent in the region of $2 N_{c} / g^{2} \simeq 2 N_{c}$ for color $S U(2)$ [23] and $S U(3)$ [20]. For color $S U(3)$, the MC simulations indicate that the critical coupling $\beta_{c}$ at $\mu=0$ seems to be a smooth function of $T=1 / N_{\tau}[10,51,76,81-$ $83,85]$ and reaches $\beta=5.08$ for $N_{\tau}=8$ [83]. When we take into account the NNLO contributions in SC-LQCD with quarks for color $S U(3), T_{c, \mu=0}$ and $\mu_{c, T=0}$ are found to be very similar to those in NLO in the region $\beta \leq 6$ [90]. These observations suggest that the strong coupling expansion does not break down in the region $\beta \leq 6$. It would be necessary to investigate the NNLO effects on the critical point and PCR matter in order to examine the present results.

There are several points to be discussed further. When we take into account NNLO contributions, the Polyakov loop can appear from two plaquettes. Hence it becomes possible to investigate the phase transitions with three order parameters, $\sigma, \rho_{q}$, and the Polyakov loop. In addition, the Polyakov loop contributions in the NNLO may modify the $\beta$ dependence of $T_{c}$. The higher order of the $1 / d$ expansion is also an important subject to be studied. The baryonic contributions are included in the subleading order of the $1 / d$ expansion, and would be essential to solve a challenging problem: nuclear matter on the lattice.

\section{ACKNOWLEDGMENTS}

We would like to thank Philippe de Forcrand, Koichi Yazaki, and Koji Hashimoto for useful discussions. This work is supported in part by KAKENHI, under the Grants No. 17070002 and No. 19540252, the Global COE Program "The Next Generation of Physics, Spun from Universality and Emergence," and the Yukawa International Program for Quark-hadron Sciences (YIPQS).
[1] For a recent review, see B. Muller and J. L. Nagle, Annu. Rev. Nucl. Part. Sci. 56, 93 (2006).

[2] K. H. Ackermann et al. (STAR Collaboration), Phys. Rev. Lett. 86, 402 (2001); C. Adler et al. (STAR Collaboration), Phys. Rev. Lett. 87, 182301 (2001); 89, 132301 (2002); Phys. Rev. C 66, 034904 (2002); J. Adams et al. (STAR Collaboration), Phys. Rev. C 72, 014904 (2005).

[3] K. Adcox et al. (PHENIX Collaboration), Phys. Rev. Lett. 89, 212301 (2002); S.S. Adler et al. (PHENIX Collaboration), Phys. Rev. Lett. 91, 182301 (2003).

[4] B. B. Back et al. (PHOBOS Collaboration), Phys. Rev. Lett. 89, 222301 (2002); 94, 122303 (2005); Phys. Rev. C 72, 051901 (2005).

[5] For a recent review, see U. M. Heller, Proc. Sci., LAT2006 (2006) 011; S. Ejiri, Nucl. Phys. B, Proc. Suppl. 94, 19 (2001).

[6] For a recent review, see O. Philipsen, Proc. Sci., LAT2005 (2006) 016; P. Petreczky, Nucl. Phys. A785, 10 (2007); Nucl. Phys. B, Proc. Suppl. 140, 78 (2005); E. Laermann and O. Philipsen, Annu. Rev. Nucl. Part. Sci. 53, 163 (2003); F. Karsch, Nucl. Phys. A698, 199 (2002); Lect. Notes Phys. 583, 209 (2002).

[7] For a recent review, see S. Muroya, A. Nakamura, C. Nonaka, and T. Takaishi, Prog. Theor. Phys. 110, 615 (2003); F. Karsch, Prog. Theor. Phys. Suppl. 153, 106 (2004), and references therein.

[8] C. R. Allton, M. Doring, S. Ejiri, S. J. Hands, O.
Kaczmarek, F. Karsch, E. Laermann, and K. Redlich, Phys. Rev. D 71, 054508 (2005); K. Redlich, F. Karsch, and A. Tawfik, J. Phys. G 30, S1271 (2004).

[9] P. de Forcrand and O. Philipsen, Nucl. Phys. B642, 290 (2002); B673, 170 (2003).

[10] M. D'Elia and M. P. Lombardo, Phys. Rev. D 67, 014505 (2003).

[11] S. Kratochvila and P. de Forcrand, Proc. Sci., LAT2005 (2005) 167; A. Li, X. Meng, A. Alexandru, and K. F. Liu, Proc. Sci., LAT2008 (2008) 178.

[12] Z. Fodor and S. D. Katz, J. High Energy Phys. 03 (2002) 014.

[13] Z. Fodor, S. D. Katz, and C. Schmidt, J. High Energy Phys. 03 (2007) 121.

[14] M. Asakawa and K. Yazaki, Nucl. Phys. A504, 668 (1989); M. A. Stephanov, K. Rajagopal, and E. V. Shuryak, Phys. Rev. Lett. 81, 4816 (1998).

[15] S. Ejiri, Phys. Rev. D 77, 014508 (2008).

[16] S. Aoki et al. (WHOT-QCD Collaboration), Proc. Sci., LAT2008 (2008) 189.

[17] C. R. Allton, S. Ejiri, S. J. Hands, O. Kaczmarek, F. Karsch, E. Laermann, and C. Schmidt, Phys. Rev. D 68, 014507 (2003); C. R. Allton et al., Phys. Rev. D 71, 054508 (2005).

[18] P. de Forcrand and O. Philipsen, J. High Energy Phys. 11 (2008) 012

[19] For a review, see I. Montvay and G. Münster, Quantum 
Fields on a Lattice (Cambridge University Press, Cambridge, 1994).

[20] For a review, see J. M. Drouffe and J. B. Zuber, Phys. Rep. 102, 1 (1983).

[21] K. G. Wilson, Phys. Rev. D 10, 2445 (1974).

[22] M. Creutz, Phys. Rev. D 21, 2308 (1980); M. Creutz and K. J. M. Moriarty, Phys. Rev. D 26, 2166 (1982).

[23] G. Münster, Nucl. Phys. B180, 23 (1981).

[24] E. Seiler, Lect. Notes Phys. 159, 1 (1982); K. Osterwalder and E. Seiler, Ann. Phys. (N.Y.) 110, 440 (1978).

[25] N. Kawamoto and J. Smit, Nucl. Phys. B192, 100 (1981).

[26] J. Hoek, N. Kawamoto, and J. Smit, Nucl. Phys. B199, 495 (1982).

[27] N. Kawamoto and K. Shigemoto, Phys. Lett. 114B, 42 (1982); Nucl. Phys. B237, 128 (1984).

[28] J. Smit, Nucl. Phys. B175, 307 (1980).

[29] H. Kluberg-Stern, A. Morel, O. Napoly, and B. Petersson, Nucl. Phys. B190, 504 (1981).

[30] H. Kluberg-Stern, A. Morel, and B. Petersson, Nucl. Phys. B215, 527 (1983).

[31] T. Jolicoeur, H. Kluberg-Stern, M. Lev, A. Morel, and B. Petersson, Nucl. Phys. B235, 455 (1984).

[32] H. Kluberg-Stern, A. Morel, O. Napoly, and B. Petersson, Nucl. Phys. B220, 447 (1983).

[33] I. Ichinose, Phys. Lett. 135B, 148 (1984); Nucl. Phys. B249, 715 (1985).

[34] P. H. Damgaard, N. Kawamoto, and K. Shigemoto, Phys. Rev. Lett. 53, 2211 (1984); Nucl. Phys. B264, 1 (1986).

[35] P. H. Damgaard, D. Hochberg, and N. Kawamoto, Phys. Lett. 158B, 239 (1985).

[36] G. Fäldt and B. Petersson, Nucl. Phys. B265, 197 (1986).

[37] N. Bilic, K. Demeterfi, and B. Petersson, Nucl. Phys. B377, 651 (1992).

[38] N. Bilic, F. Karsch, and K. Redlich, Phys. Rev. D 45, 3228 (1992).

[39] N. Bilic and J. Cleymans, Phys. Lett. B 355, 266 (1995).

[40] Y. Nishida, K. Fukushima, and T. Hatsuda, Phys. Rep. 398, 281 (2004).

[41] V. Azcoiti, G. Di Carlo, A. Galante, and V. Laliena, J. High Energy Phys. 09 (2003) 014.

[42] K. Fukushima, Prog. Theor. Phys. Suppl. 153, 204 (2004).

[43] Y. Nishida, Phys. Rev. D 69, 094501 (2004).

[44] N. Kawamoto, K. Miura, A. Ohnishi, and T. Ohnuma, Phys. Rev. D 75, 014502 (2007).

[45] K. Miura, N. Kawamoto, and A. Ohnishi, Proc. Sci., LAT2008 (2008) 075.

[46] K. Miura, T.Z. Nakano, and A. Ohnishi, Prog. Theor. Phys. 122, 1045 (2009); A. Ohnishi and K. Miura, Proc. Sci., LAT2008 (2008) 192.

[47] A. Ohnishi, N. Kawamoto, and K. Miura, Mod. Phys. Lett. A 23, 2459 (2008); N. Kawamoto, K. Miura, and A. Ohnishi, Prog. Theor. Phys. Suppl. 174, 250 (2008); Proc. Sci., LAT2007 (2007) 209.

[48] A. Ohnishi, N. Kawamoto, K. Miura, K. Tsubakihara, and H. Maekawa, Prog. Theor. Phys. Suppl. 168, 261 (2007).

[49] A. Ohnishi, N. Kawamoto, and K. Miura, J. Phys. G 34, S655 (2007).

[50] B. Bringoltz, J. High Energy Phys. 03 (2007) 016.

[51] P. de Forcrand and M. Fromm, arXiv:0907.1915.

[52] E. Dagotto, A. Moreo, and U. Wolff, Phys. Rev. Lett. 57, 1292 (1986); Phys. Lett. B 186, 395 (1987).
[53] F. Karsch and K.H. Mütter, Nucl. Phys. B313, 541 (1989).

[54] A. R. Levi, V. Lubicz, and C. Rebbi, Phys. Rev. D 56, 1101 (1997).

[55] Y. Umino, Phys. Lett. B 492, 385 (2000); Mod. Phys. Lett. A 17, 2513 (2002); Phys. Rev. D 66, 074501 (2002); Mod. Phys. Lett. A 21, 55 (2006).

[56] Y.Z. Fang and X. Q. Luo, Int. J. Mod. Phys. A 16, 4499 (2001).

[57] X. Q. Luo, Phys. Rev. D 70, 091504(R) (2004).

[58] R. C. Brower and B. Svetitsky, Phys. Rev. D 61, 114511 (2000).

[59] L. Levkova and R. Mawhinney, Nucl. Phys. B, Proc. Suppl. 140, 695 (2005).

[60] I. Ichinose and K. Nagao, Nucl. Phys. B577, 279 (2000); B596, 231 (2001).

[61] P. Ye, X. L. Yu, Y. Guan, and X. Q. Luo, Mod. Phys. Lett. A 22, 547 (2007); X. L. Yu and X. Q. Luo, Mod. Phys. Lett. A 22, 537 (2007).

[62] P. Hasenfratz and F. Karsch, Phys. Lett. 125B, 308 (1983).

[63] J. Polonyi and K. Szlachanyi, Phys. Lett. 110B, 395 (1982); M. Gross, J. Bartholomew, and D. Hochberg, Report No. EFI-83-35-CHICAGO, 1983.

[64] E. M. Ilgenfritz and J. Kripfganz, Z. Phys. C 29, 79 (1985).

[65] A. Gocksch and M. Ogilvie, Phys. Rev. D 31, 877 (1985).

[66] K. Fukushima, Phys. Lett. B 553, 38 (2003); Phys. Rev. D 68, 045004 (2003).

[67] K. Fukushima, Phys. Lett. B 591, 277 (2004).

[68] J. Langelage, G. Münster, and O. Philipsen, J. High Energy Phys. 07 (2008) 036.

[69] A. Jakovac and D. Nogradi, arXiv:0810.4181.

[70] D. B. Kaplan, Phys. Lett. B 288, 342 (1992).

[71] H. Neuberger, Phys. Lett. B 427, 353 (1998).

[72] L. Susskind, Phys. Rev. D 16, 3031 (1977).

[73] H. S. Sharatchandra, H. J. Thun, and P. Weisz, Nucl. Phys. B192, 205 (1981).

[74] M.F. L. Golterman and J. Smit, Nucl. Phys. B245, 61 (1984); B255, 328 (1985); M. F. L. Golterman, Nucl. Phys. B273, 663 (1986); B278, 417 (1986).

[75] For a recent review, see the first half of A. S. Kronfeld, Proc. Sci., LAT2007 (2007) 016; S. R. Sharpe, Proc. Sci., LAT2006 (2006) 022.

[76] Z. Fodor and S. D. Katz, Phys. Lett. B 534, 87 (2002).

[77] M. Creutz, J. Math. Phys. (N.Y.) 19, 2043 (1978); I. Bars, J. Math. Phys. (N.Y.) 21, 2678 (1980); S. Samuel, J. Math. Phys. (N.Y.) 21, 2695 (1980); K.E. Eriksson, N. Svartholm, and B.S. Skagerstam, J. Math. Phys. (N.Y.) 22, 2276 (1981); J. Hoek, Phys. Lett. 102B, 129 (1981); R. C. Brower and M. Nauenberg, Nucl. Phys. B180, 221 (1981); S. I. Azakov and E. S. Aliev, Phys. Scr. 38, 769 (1988); F. C. Hansen, "SU(N) Haar Measure Integrals in the Nonlinear Sigma Model," Report No. BUTP-90/42 (unpublished).

[78] M.E. Fisher and D.S. Gaunt, Phys. Rev. 133, A224 (1964).

[79] For example, R. Kubo, J. Phys. Soc. Jpn. 17, 1100 (1962).

[80] B. D. Serot and J. D. Walecka, Adv. Nucl. Phys. 16, 1 (1986); K. Tsubakihara and A. Ohnishi, Prog. Theor. Phys. 117, 903 (2007).

[81] P. de Forcrand (private communication). 
[82] S. A. Gottlieb, W. Liu, D. Toussaint, R. L. Renken, and R. L. Sugar, Phys. Rev. D 35, 3972 (1987).

[83] R. V. Gavai et al. (MT(c) Collaboration), Phys. Lett. B 241, 567 (1990).

[84] A. D. Kennedy, J. Kuti, S. Meyer, and B. J. Pendleton, Phys. Rev. Lett. 54, 87 (1985).

[85] G. Boyd, J. Fingberg, F. Karsch, L. Karkkainen, and B. Petersson, Nucl. Phys. B376, 199 (1992).

[86] L. McLerran and R. D. Pisarski, Nucl. Phys. A796, 83 (2007); Y. Hidaka, L. D. McLerran, and R. D. Pisarski, Nucl. Phys. A808, 117 (2008); L. McLerran, K. Redlich, and C. Sasaki, Nucl. Phys. A824, 86 (2009).

[87] K. Fukushima, Phys. Rev. D 77, 114028 (2008); 78,
039902(E) (2008); H. Abuki, R. Anglani, R. Gatto, G. Nardulli, and M. Ruggieri, Phys. Rev. D 78, 034034 (2008).

[88] M. Kitazawa, T. Koide, T. Kunihiro, and Y. Nemoto, Prog. Theor. Phys. 108, 929 (2002); C. Sasaki, B. Friman, and K. Redlich, Phys. Rev. D 75, 054026 (2007); K. Fukushima, Phys. Rev. D 78, 114019 (2008).

[89] R. D. Pisarski and F. Wilczek, Phys. Rev. D 29, 338 (1984).

[90] A. Ohnishi, K. Miura, and T.Z. Nakano, Proc. Sci. (to be published); T. Z. Nakano, K. Miura, and A. Ohnishi (unpublished). 